\title{
Evidence of a fast evolution of the UV luminosity function beyond redshift 6 from a deep HAWK-I survey of the GOODS-S field
}

\author{
M. Castellano ${ }^{1}$, A. Fontana ${ }^{1}$, K. Boutsia ${ }^{1}$, A. Grazian ${ }^{1}$, L. Pentericci ${ }^{1}$, R. Bouwens ${ }^{2}$, M. Dickinson ${ }^{3}$, M. Giavalisco ${ }^{4}$, \\ P. Santini ${ }^{1}$, S. Cristiani ${ }^{5}$, F. Fiore ${ }^{1}$, S. Gallozzi ${ }^{1}$, E. Giallongo ${ }^{1}$, R. Maiolino ${ }^{1}$, F. Mannucci ${ }^{6}$, N. Menci ${ }^{1}$,

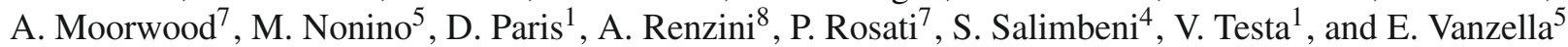 \\ 1 INAF - Osservatorio Astronomico di Roma, via Frascati 33, 00040 Monteporzio (RM), Italy \\ e-mail: castellano@oa-roma.inaf.it \\ 2 Lick Observatory, University of California, Santa Cruz, CA 95064, USA \\ NOAO, 950 N. Cherry Avenue, Tucson, AZ 85719, USA \\ 4 Department of Astronomy, University of Massachusetts, 710 North Pleasant Street, Amherst, MA 01003, USA \\ 5 INAF - Osservatorio Astronomico di Trieste, via G.B. Tiepolo 11, 34131 Trieste, Italy \\ 6 INAF - Osservatorio Astrofisico di Arcetri, Largo E. Fermi 5, 50125 Firenze, Italy \\ 7 European Southern Observatory, Karl-Schwarzschild-Str. 2, 85748 Garching, Germany \\ 8 INAF - Osservatorio Astronomico di Padova, Vicolo dell'Osservatorio 5, 35122 Padova, Italy
}

Received 15 September 2009 / Accepted 10 November 2009

\section{ABSTRACT}

\begin{abstract}
Aims. We perform a deep search for galaxies in the redshift range $6.5 \leq z \leq 7.5$, to measure the evolution of the number density of luminous galaxies in this redshift range and derive useful constraints on the evolution of their luminosity function.

Methods. We present here the first results of an ESO Large Programme, which exploits the unique combination of area and sensitivity provided in the near-IR by the camera Hawk-I at the VLT. We have obtained two Hawk-I pointings on the GOODS South field for a total of $\sim 32$ observing hours, covering $\sim 90 \operatorname{arcmin}^{2}$. The images reach $Y=26.7$ mag for the two fields. We used public ACS images in the $z$ band to select $z$-dropout galaxies with the colour criteria $Z-Y \geq 1, Y-J<1.5$, and $Y-K<2$. The other public data in the UBVRIJK bands are used to reject possible low redshift interlopers. The output has been compared with extensive Monte Carlo simulations to quantify the observational effects of our selection criteria, as well as the effects of photometric errors.

Results. We detect 7 high-quality candidates in the magnitude range $Y=25.5-26.7$. This interval samples the critical range for $M_{*}$ at $z>6\left(M_{1500} \simeq-19.5\right.$ to -21.5$)$. After accounting for the expected incompleteness, we rule out a luminosity function constant from $z=6$ to $z=7$ at a $99 \%$ confidence level, even including the effects of cosmic variance. For galaxies brighter than $M_{1500}=-19.0$, we derive a luminosity density $\rho_{\mathrm{UV}}=1.5_{-0.9}^{+2.0} \times 10^{25} \mathrm{erg} \mathrm{s}^{-1} \mathrm{~Hz}^{-1} \mathrm{Mpc}^{-3}$, implying a decrease by a factor 3.5 from $z=6$ to $z \simeq 6.8$. On the basis of our findings, we make predictions for the surface densities expected in future surveys, based on ULTRA-VISTA, HST-WFC3, or JWST-NIRCam, evaluating the best observational strategy to maximise their impact.
\end{abstract}

Key words. galaxies: distances and redshifts - galaxies: high-redshift - galaxies: luminosity function, mass function galaxies: evolution

\section{Introduction}

The search for extremely high-redshift galaxies is much more than an exciting exploration of the furthest frontiers of the Universe, although this aspect is certainly a reason for its popularity. Its actual astrophysical interest is tied to the constraints that can be set on the physical mechanisms that drove the formation and evolution of galaxies at the earliest epochs of the Universe.

One important area of interest in the study of galaxies at $z>6$ is ascertaining their role in the reionisation of the Universe. To be fully responsible for the reionisation, the density of starforming galaxies at $z>7$ should have been similar to that at $z \simeq 4$, unless there is a significant evolution in the IMF and/or in the clumpiness of the IGM, in the escape fraction of ionising photons or in their metallicity (see e.g. Mannucci et al. 2007; Oesch et al. 2009, and references therein). Quantifying their number density is therefore critical for constraining the additional mechanisms that may be responsible for the re-ionisations, like Pop III dominated primordial galaxies, mini-black holes, or others (see e.g. Venkatesan et al. 2003; Madau et al. 2004).

Understanding the evolution of galaxies at high redshift is also very important in the broader context of galaxy evolution. While modelling the growth of structures of dark matter is relatively straightforward, modelling the physical mechanisms of star formation and feedback that shaped galaxies across the life of the Universe from first principles is remarkably complex.

The fundamental quantity that is currently used to describe and quantify the galaxy population at high redshift is the UV luminosity function (LF hereafter), as derived from surveys of Lyman break galaxies (LBGs) at various redshifts. The evolution of its shape and normalisation along the cosmic time provides a clear picture of the evolution of star-forming galaxies in the early Universe, and an important constraint on the related theoretical predictions.

Searches for LBGs have been extremely successful out to redshift 6 (e.g. Steidel et al. 1995, 1999; Bunker et al. 2004; Dickinson et al. 2004; Giavalisco et al. 2004; Ouchi et al. 2004; 
Yoshida et al. 2006; Bouwens et al. 2003, 2006, 2007; McLure et al. 2009) i.e. when the Universe was only less than 1 Gyr old. Current samples of high-redshift galaxies $(z \sim 3-6)$ now contain tens of thousands of galaxies and extend to luminosities as faint as $-16 \mathrm{AB}$ mag $\left(0.01 L^{*}\right)$.

Despite these large samples, there is still controversy on how the UV LF evolves at high-redshift. Some authors (Sawicki \& Thompson 2006; Iwata et al. 2007) have argued that the most significant evolution in the UV LF happens at the faint end, others (Yoshida et al. 2006; Bouwens et al. 2006, 2007) find that the most significant evolution is at the bright end, while Beckwith et al. (2006) claim that the evolution is similar at the bright and faint ends. Other authors suggest compensating evolutions in LBG number density and characteristic luminosity, resulting in a nearly constant UV luminosity density (Dickinson et al. 2004; Giavalisco et al. 2004). The likely origins of these discrepancies are both the strong effect of cosmic variance (see e.g. Trenti \& Stiavelli 2008) and systematic effects of the different estimates of completeness level, contamination from lower redshift interlopers, volume elements, and redshift distributions in the various samples (Stanway et al. 2008a), all worsened by the known degeneracy among the parameters adopted to fit the LF. A clear example of the effect of these uncertainties is shown by the recent revision of the estimated slope of the UV LF at $z=2-3$, where LBG samples are bigger and carefully characterised (Reddy \& Steidel 2009).

Even within these uncertainties, it is becoming clear that the overall evolution of the UV LF in the redshift range $z=2-6$ implies a decrease in the number density of UV bright galaxies $(M<-20.5)$ of a factor 6-11 from $z \sim 3$ to $z \sim 6$ (e.g. Stanway et al. 2003; Shimasaku et al. 2005; Bouwens et al. 2006).

On the other hand, our knowledge of the evolution beyond $z \sim 6$ is much more scanty. Finding and studying galaxies at $z=7$ to constrain the UV LF is definitely challenging, requiring deep and wide surveys in the near-IR part of the spectrum. Currently, the only constraints come from a small sample of faint $z \sim 7-9$ candidates found in small areas within GOODS with deep near-IR $J+H$ NICMOS and WFC3 data (Bouwens \& Illingworth 2006; Bouwens et al. 2008; Oesch et al. 2009; Bouwens et al. 2010; Oesch et al. 2010; McLure et al. 2010; Bunker et al. 2009). Dropout searches around lensing clusters have also been performed, (Richard et al. 2006, 2008; Bradley et al. 2008; Bouwens et al. 2009; Zheng et al. 2009). The discrepant results of these lensing studies clearly highlight the difficulties in detecting $z>6$ candidates and in removing interlopers from such samples (see Bouwens et al. 2009, for a discussion).

Further constraints come from the lack of bright $z>6.5$ candidate galaxies in relatively wide and shallower observation (Mannucci et al. 2007; Henry et al. 2009). Spectroscopic identifications of $z>6.5$ LBGs are lacking until now, with the exception of the narrow-band selected Ly $\alpha$ emitter at $z=6.96$ by Iye et al. (2006).

Apart from some contradictory results around lensing clusters, all the evidence suggests that the number density of UV-bright galaxies fades significantly at $z>6.5$, amounting to a decrease of the volume density at the bright end of the UV LF of a factor 10-30 from $z \sim 4$ to $z \sim 7$ (Mannucci et al. 2007; Bouwens et al. 2007; Stanway et al. 2008b; Oesch et al. 2009). It is indeed possible to use these observations to constrain the standard Schechter parameters of the LF, which appear to deviate significantly from the $z=6$ ones, although the statistical uncertainties are embarrassingly large. If one takes also into account the many uncertainties due to systematic error in the candidate selection and cosmic variance, it becomes clear that the evolution of the LF at $z>6$ is still largely unexplored.

To progress in this field, larger and deeper IR-based surveys are definitely needed. The very recent WFC3 data are providing a dramatic advance, accessing the faint side of the LF at $z \simeq 7$ and extending the searches to $z \simeq 10$ (Bouwens et al. 2010; Oesch et al. 2010; McLure et al. 2010; Bunker et al. 2009; Yan et al. 2009). In parallel, we are conducting a search of $z \simeq 7$ bright galaxies on wider areas using the new VLT IR imager Hawk-I (Pirard et al. 2004; Casali et al. 2006; Kissler-Patig et al. 2008), which is complementary to it in many respects. First, we can cover significantly larger areas, albeit shallower than WFC3, thanks to the wide field-of view of Hawk-I. This will yield a statistically adequate sampling at the brightest magnitudes, which is needed to obtain an accurate estimate of the LF parameters.

In addition, we use the $Y$ band to detect galaxies at $z>6.5$, instead of the $J$ band used so far. Thanks to the lower sky background and to the extreme efficiency of Hawk-I, it is possible to reach the required faint limits (close to $\simeq 27 \mathrm{mag} A B$ ). Such faint magnitudes can be easily reached in the $J$ band only from space. More important, the shorter central wavelength (around $1 \mu \mathrm{m}$ ) of the $Y$ filter corresponds to a lower and narrower redshift range, roughly $6.4<z<7.2$ (see Sect. 4 and Fig. 7). This fair sampling is very important to verify whether the UV LF evolves quickly at $z>6$.

In this paper we discuss the results of the first half of our survey, covering a large fraction of the GOODS-S field. The paper is organised as follows. In Sect. 2 we present our data set and the multi-wavelength catalogue; in Sect. 3 we discuss our colour selection criteria and the potential interlopers affecting the LBG selection; in Sect. 4 we evaluate systematic effects in the light of extensive Monte Carlo simulations; in Sect. 5 we present our final sample of candidate $z$-drop LBGs, which is used to constrain the evolution of the $z>6$ UV LF in Sect. 6. In Sect. 7 we discuss the implications of our findings, of the present uncertainties on the high- $z$ LF, and on the efficiency of future dedicated surveys. A summary of our methods and results is provided in Sect. 8.

Throughout the whole paper, observed and rest-frame magnitudes are in the $\mathrm{AB}$ system, and we adopt the $\Lambda$-CDM concordance model $\left(H_{0}=70 \mathrm{~km} \mathrm{~s}^{-1} \mathrm{Mpc}^{-1}, \Omega_{\mathrm{M}}=0.3\right.$, and $\left.\Omega_{\Lambda}=0.7\right)$.

\section{Data}

\subsection{The data set}

This work is based on deep $Y$-band images obtained with Hawk-I, the new near-IR camera installed at the VLT. With a field of view of about $7.5^{\prime} \times 7.5^{\prime}$, pixel scale of $0.1^{\prime \prime}$, excellent sensitivity and image quality, it is currently one of the best ground-based instruments for searching for faint, rare objects as very high-redshift galaxies.

We combine data collected in 2007 during the Hawk-I Science Verification phase with further images obtained in 2008 through a dedicated ESO Large Programme. The images cover two adjacent regions of the GOODS-S field, corresponding to $\sim 85 \%$ of the deep ACS area. We name these two pointing GOODS1 (southern) and GOODS2 (northern). The data obtained with the ESO LP extend the coverage of the GOODS2 field (originally about $11 \mathrm{hrs}$ in the $Y$ band) to the depth of the GOODS1 area. The total exposure time is $16 \mathrm{~h} 15 \mathrm{~m}$ for GOODS1 and $16 \mathrm{~h} 56 \mathrm{~m}$ for GOODS2. The position of the two combined Hawk-I fields is shown in Fig. 1. 


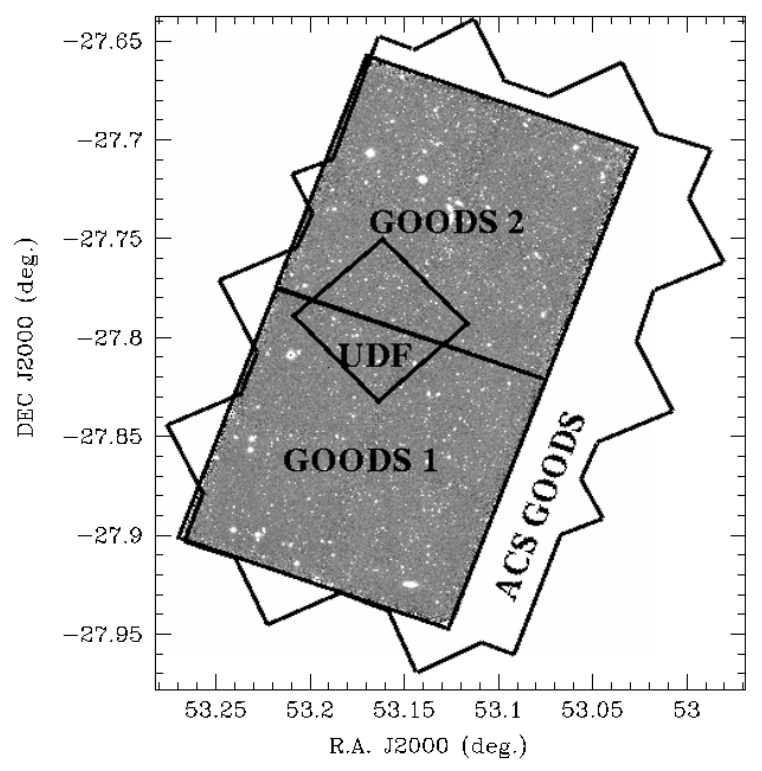

Fig. 1. Full mosaic of the two Hawk-I images of the GOODS-S field. The jigsaw region shows the original GOODS ACS $z$-band image. The position of the UDF is also shown.

The $Y$ band images were reduced using standard techniques for IR data - flat fielding, sky subtraction among consecutive frames, and final coaddition. Particular care was taken to mask even the faintest sources during the sky subtraction. This was accomplished with a "double-pass" procedure, where the faintest sources were detected in a first version of the finally coadded images, and the whole data reduction was repeated masking all objects during the sky estimate step.

Two classes of defects are present in the Hawk-I images. One class consists of luminous "ripples" along the rows originating from bright saturated objects. These defects come from crosstalk effects and have been removed by masking the whole range of affected rows in each image. Other problems are caused by a "persistence" effect, i.e. residuals left by sources which appear as faint objects on the next acquisition at the previous position (in pixel) of the bright object. These "ghosts" may appear as faint dropouts in the final image, unless some masking is done. We decided to remove these objects from the beginning, by masking (in each image) the pixels where objects were detected in the previous one $(\sim 0.3-1.0 \%$ of the total area in each science frame). This strategy turned out to be more effective and safer than just making a sigma-clipping during the final coaddition. The final images have been registered to the ACS astrometric solution, maintaining the original Hawk-I pixel size.

We find that the GOODS1 and GOODS2 fields are rather uniform in their general properties. Through the analysis of bright point-like sources, we determine an FWHM of $0.51 \pm$ $0.01 \operatorname{arcsec}(=4.8$ pixels $)$ in the GOODS1 image and $0.49 \pm$ $0.02 \operatorname{arcsec}(=4.6$ pixels) in the GOODS2 one. We computed image zeropoints using the standard stars observed during the same night of the GOODS data and at similar airmasses. Since the standard stars are calibrated on A0V stars in the MKO filter set, we estimated the conversion to the $\mathrm{AB}$ system for the Hawk-I specific $Y$ band filter using templates, as $Y_{\text {Hawk-I }}=$ $Y_{\mathrm{MKO}}+\left(J_{\mathrm{MKO}}-K_{\mathrm{MKO}}\right)$. The resulting zeropoints are $Y=26.992$ and $Y=26.998$ (AB) for GOODS1 and GOODS2 respectively.

We have also carefully determined the rms of the coadded images, which is needed to properly estimate the statistical meaning of detections at the faint limit. We obtained "from first principles" an absolute rms that fully accounts for the correlation in the pixel of the finally coadded image. This was estimated by computing the rms in each individual image (using the Poisson statistics and the instrumental gain) and propagating self-consistently this rms over the whole data reduction process. The typical $5 \sigma$ magnitude in one $\operatorname{arcsec}^{2}$ is in the range 26.7-26.8 over more than $60 \%$ of the whole image, and $>26.2$ in $85 \%$ of the image - the rest of the images being shallower because of the gaps between the four Hawk-I chips.

\subsection{The photometric catalogue - detection}

We have obtained the photometric catalogue using the SExtractor code (Bertin \& Arnouts 1996) and the $Y$ band as detection image. The rms derived "from first principles" as described above is used in SExtractor as MAP_RMS and overrides the rms obtained by SExtractor from the background fluctuations. To obtain total magnitudes, we have computed both the SExtractor's MAG_BEST and aperture corrected magnitudes in 2 FWHM diameter (about $1^{\prime \prime}$ ), the same apertures used for the colour estimate discussed below. We computed the aperture corrections from bright non-saturated stars in each field: we find corrections of $0.364 \mathrm{mag}$ and $0.322 \mathrm{mag}$ in GOODS 1 and GOODS2, respectively. MAG_BEST magnitudes are more accurate for bright objects, but become fainter than aperturecorrected ones at about $m_{Y} \simeq 24$. As we show below, all $z>6.5$ candidates are so faint that their total magnitude is estimated with aperture-corrected magnitudes. For resolved objects, however, aperture corrections based on stellar profile may underestimate the actual total flux. We have estimated this effect by using LBGs with known spectroscopic redshifts $5.5<z<6.2$ in the GOODS-S ACS images (Vanzella et al. 2009), smoothing them to the Hawk-I PSF. We find that the aperture correction is slightly larger, i.e. $\sim 0.5$. For simplicity, and given the unknown physical extent of our $z>6.5$ galaxies, we shall adopt the nominal aperture correction based on known stars for all candidates. However, the effect of lost flux due to the finite size of our candidates is fully accounted for by the simulations that we use to estimate the LF, as we discuss in Sect. 4.

The most critical issue is the very detection at faint limits. Given the extreme faintness expected for the $z>6.5$ galaxies and their rarity, a compromise must be found between two competing goals: extending the detection at the faintest possible levels while retaining good accuracy. In addition, the systematics must be understood and quantified for the final scientific analysis. We optimised the SExtractor parameters involved in the detection of faint objects evaluating at the same time the possible contamination from spurious objects in the $Y$-detected catalogue through the analysis of a "negative" image. To do this, we varied the detection parameters used by SExtractor (DETECT_MINAREA, DETECT_THRESH, filtering, deblending, and background subtraction parameters) in the analysis of the "positive" image. We then constructed a "background-subtracted negative" image and analysed it exactly as the positive one, with the same SExtractor parameters. The rms image used is the same employed in the analysis of the "positive" image. We finally adopted the set of parameters that minimises the ratio between "negative" and "positive" detections at the faint end of the number counts. The final detection is obtained requiring 10 contiguous pixels each at $S / N>0.727$, corresponding to a $2.3 \sigma$ detection, and restricting the analysis to the regions where the rms is less than 1.5 times the lowest value. With this choice of parameters, detections on the negative images are negligible at $Y<26.5$, and less than $10 \%$ down to $Y=26.8$ in both fields. However, a posteriori, the 
latter value overestimates the actual rate of spurious detections. Indeed, all spurious sources should appear as "drop-out" candidates with a single-band detection. On the contrary, as we show in Sect. 5, our two faintest candidates are both confirmed by detections in other IR bands. A visual inspection of the negative images shows that many faint "objects" are found near bright sources, and this is an indication that, at the faintest limits, nontrivial issues concerning the subtraction of the background or a potential asymmetry in the noise distribution produce an overestimate of the rate of spurious detections.

This procedure is also used to define the total area where a homogeneous catalogue can be extracted. The candidates found in this area will be used for the evaluation of the LF. This area is $\sim 75 \%$ of the whole image and is $89.7 \operatorname{arcmin}^{2}$. We point out that additional candidates satisfying our colour and $S / N$ selection criteria (see Sect. 3) are also found in the noisier regions of the images, the most notable being an object falling in the UDF, but they will not be discussed in this paper.

\subsection{The multicolour catalogue}

We have obtained full multiwavelength photometry of all the objects detected on the $Y$ band using the publicly available UBVRIZJHK images. ACS BVIZ images are the latest V2.0 version released by the STSci (Giavalisco \& the GOODS Team, in preparation). Each frame was smoothed to the Hawk-I PSF using an appropriate kernel obtained with Fourier transform (Grazian et al. 2006) and registered to the Hawk-I images. Publicly available $U$ and $R$ images obtained with VIMOS (Nonino et al. 2009) and JHK mosaics (Retzlaff et al., in preparation) were also registered to the Hawk-I images. These have not been filtered, since their PSF is always somewhat larger than the $Y$ one. We then compute magnitudes in $U, B, V, R, I, z$, $J, H$, and $K$ s bands running SExtractor in dual mode using the $Y$ band HAWK-I image as the detection image with the detection parameters indicated above. Aperture fluxes were computed with the same aperture as in the $Y$ band, and appropriate aperture corrections were separately applied to each band. A comparison between ACS and ISAAC total magnitudes in our catalogue and in the GOODS-MUSIC catalogue (Grazian et al. 2006; Santini et al. 2009) shows good agreement, apart from a fraction of the blended objects $(10 \%$ of the total) that are unresolved in the $Y$ band detection but have been deblended in the ACS Z-detected sample of the GOODS-MUSIC catalogue. The typical $1 \sigma$ limiting magnitudes in these images, scaled to the total $Y$ band flux of detected objects (i.e. estimated in the $1.2^{\prime \prime}$ aperture and corrected to total) are 29.1, 29.0, 29.1, 29.5, 28.6, 28.2, 26.6, 26.2, 26.2 in $U, B, V, R, I, Z, J, H, K$ respectively.

This catalogue contains self-consistent magnitudes in all bands. To exploit the superior image quality of the ACS images, we have also obtained photometry of all objects on the BVIZ images without smoothing them to the Hawk-I PSF, in a narrower aperture of $0.6^{\prime \prime}$ (an even smaller aperture would be prone to errors on object centring).

\section{The selection of $z>6.5$ galaxies}

\subsection{The colour selection criterion}

The selection of galaxies at $z>6.5$ uses the well known "dropout" or "Lyman-break" technique, with minor modifications due to our filter set and imaging depth. The main spectral feature that enables the identification of galaxies at extreme redshifts is the sharp drop shortward of the Lyman- $\alpha$, where most of the photons

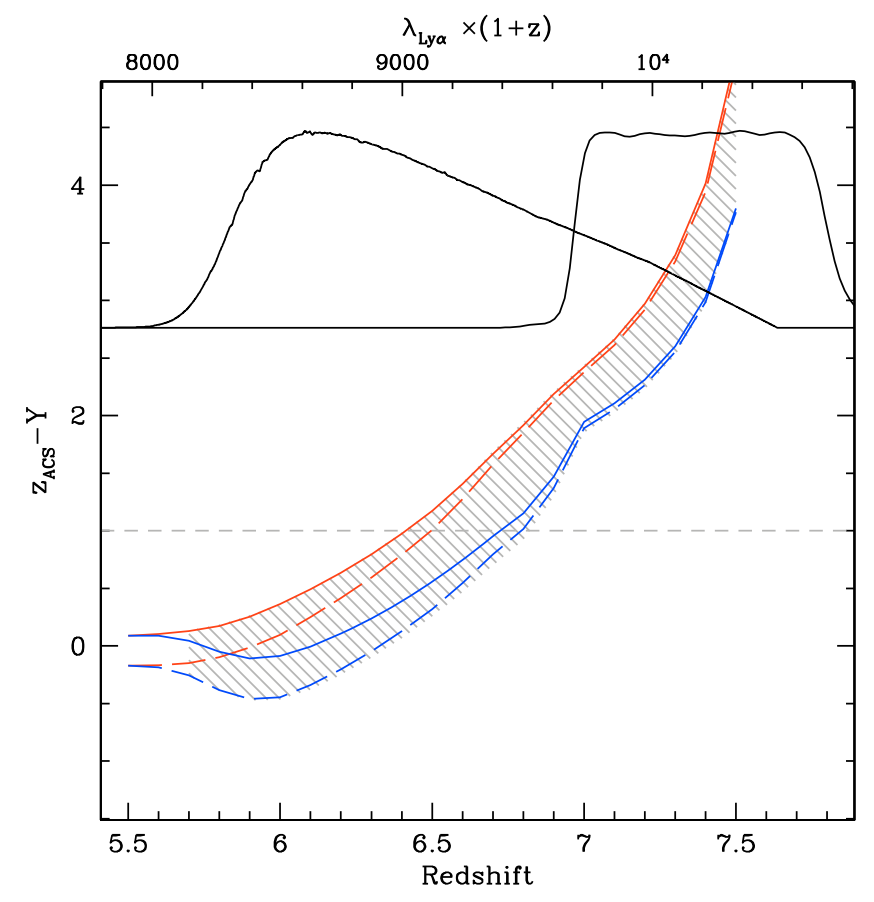

Fig. 2. $Z-Y$ colour as a function of redshift in the Hawk-I filter set. Shaded area shows the locus predicted by CB07 models with a range of metallicities, ages, dust extinction and Lyman- $\alpha$ emission (see text for details). Red lines corresponds to extreme models with no Lyman- $\alpha$, blue lines with a rest frame Lyman- $\alpha$ equivalent width of $300 \AA$. In the upper part, the efficiency curve of the two filters is shown, computed at observed wavelength of a Lyman- $\alpha$ emission at the corresponding redshift.

are absorbed by the intervening $\mathrm{HI}$ in the intergalactic medium. At $6.5<z<7.5$, this break is sampled by the large $Z-Y$ colour, as shown in Fig. 2, where we plot the $Z-Y$ colour of galaxies at $z>6$ in our filter set. At this purpose we have used the models of Charlot and Bruzual 2007 (in preparation, see Bruzual 2007a,b, hereafter CB07) with the following range of free parameters: metallicity: $0.02,0.2$ and $1 Z_{\odot}$; age from 0.01 Gyr to the maximal age of the Universe at a given $z ; E(B-V)=0 \ldots 0.2$ (Calzetti et al. 2000). Since Lyman- $\alpha$ emission has a strong influence on the selection function of LBGs, as proven for lower redshift samples (e.g. Stanway et al. 2008a; Dow-Hygelund et al. 2007), we explicitly take this effect into account by considering a distribution of Ly- $\alpha$ rest-frame equivalent width in the range 0-300 A. We also added the intergalactic absorption using the average evolution as in Madau (1995). The shaded area shows the region covered by the computed models. It is evident that the colour evolution is relatively smooth, mainly because of the extended red tail of the $Z$ filter, which gathers a fraction of flux from galaxies up to $z \simeq 7.5$.

Most of the broadening in the $Z-Y$ colour distribution of the model galaxies in Fig. 2 comes from the effect of the Lyman$\alpha$ emission line, which may change the observed colour (at a given redshift) by nearly $Z-Y \simeq 1 \mathrm{mag}$. The uncertainty in the intensity of this line can be translated into an uncertainty in the estimated redshift, which is (even ignoring noise effects) about $\Delta z \simeq 0.2-0.4$ in the redshift range $6.5<z<7.5$. Despite this, we adopt a single threshold $Z-Y \geq 1$ to select candidates.

The major difference with the standard Lyman break technique is that the $Y$ band that we use to detect galaxies does not sample the continuum around $1500 \AA$ but a region shortward of it, contaminated by both the IGM absorption and by 


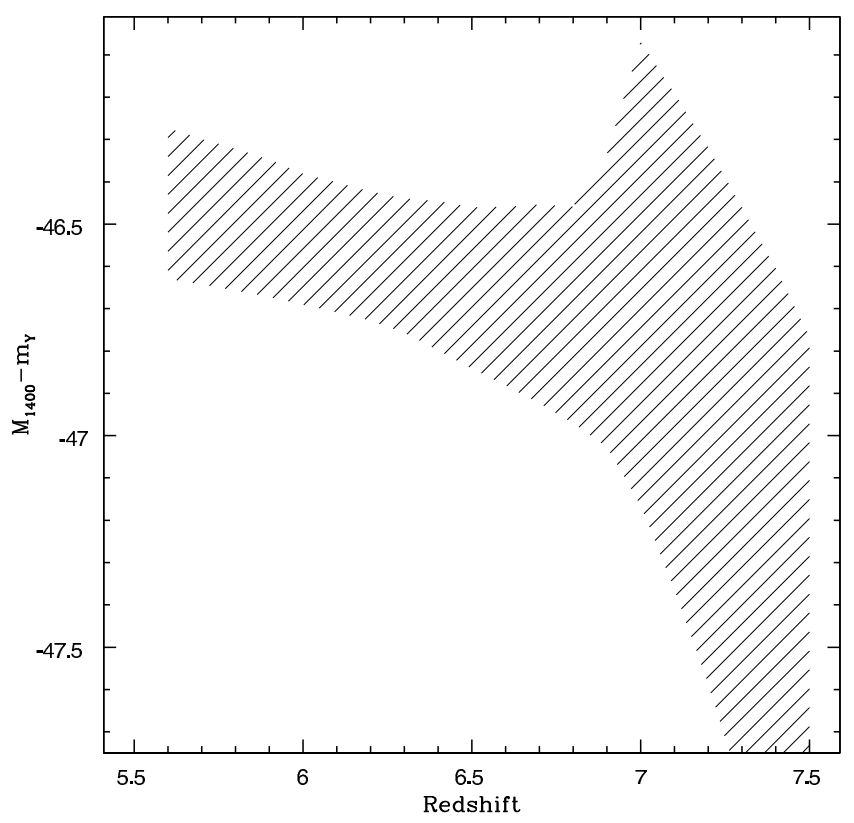

Fig. 3. The distance modulus $M_{1500}-m_{Y}$ as a function of redshift in the $Y$-band Hawk-I filter. Shaded area shows the locus predicted by CB07 models with a range of metallicities, ages, dust extinction, and Lyman- $\alpha$ emission (see text for details).

the Lyman- $\alpha$ emission line. Since we aim at comparing our candidates with those expected from the LF at $1500 \AA$, this effect can be taken into account by computing the expected distance modulus $D M(z)=M_{1500}-m_{Y}$ with the same template set described above. This is shown in Fig. 3. In addition to the cosmological dimming with redshift (for galaxies of given $M_{1500}$ ), the Lyman- $\alpha$ emission can produce a brightening at $z \geq 7$, rapidly counterbalanced at $z \geq 7.3$ by a loss of flux due to the intervening IGM absorption. All these effects are accounted for by the Monte Carlo treatment that we discuss in the next section.

\subsection{Interlopers}

Given the rarity of high-redshift galaxies, it is mandatory to discuss the possible contaminants in the selected colour criterion. Apart from $z>6$ galaxies, other well known classes of objects can display a red $Z-Y$ colour: i) variable objects, mostly due to low-intermediate redshift SN events; ii) passively evolving galaxies or dusty starburst galaxies at $z>1.5$; iii) Galactic cool stars.

We first cross-checked each object with $Z-Y>1$ against variability, by looking at images acquired at different epochs ( 1 month of delay in GOODS1, 1 year in GOODS2). We identified three obvious transients in the GOODS1 images, that have been removed from the following analysis. All the other objects in our sample have a consistent photometry in the two epochs, at the $3 \sigma$ level.

Passively evolving galaxies or dusty starburst galaxies at $z>1.5$ can be easily modelled with a suitable set of spectral synthesis models. We use the same CB07 library as for $z>5.5$ galaxies to predict the colours of such objects at $1.5<z<4$, using a combination of short star formation exponential timescales $(0.1-1 \mathrm{Gyr})$ and ages $>1$ Gyr to reproduce passively evolving galaxies, and constant star-forming models with $0.5<E(B-V)<1.5$ (adopting a Calzetti et al. 2000, extinction law) for the dusty starbursts. As shown in Fig. 4, these
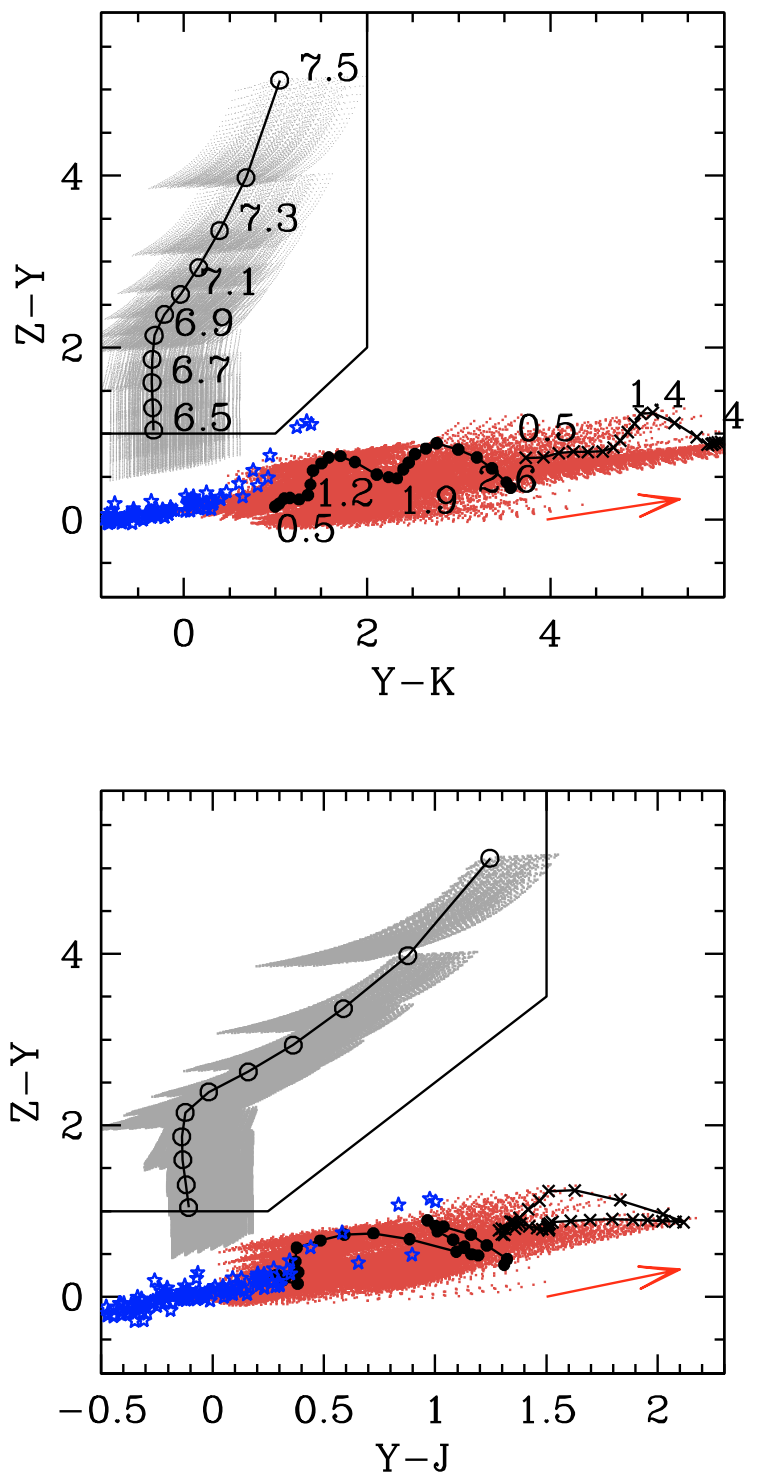

Fig. 4. Upper panel: $Z-Y-Y-K$ diagram showing the expected position of $z>6.5$ galaxies (grey dots), passively evolving galaxies and reddened starbursts (red dots) at different redshifts. See text for the details of the adopted library. The redshift evolution of a single representative model is also shown: open circles for LBGs, large filled circles for passively evolving, crosses for dusty starbursts. The red arrow represents the reddening vector at $z=1.5$. Blue symbols mark the position of normal galactic stars. Lower panel: as above, for the $Z-Y-Y-J$ colour plane.

galaxies may have large $Z-Y$ colours only when they also show large IR colour terms in the $J$ and $K$ bands. The effect of even larger amount of dust extinction would be to shift the objects at even redder $Y-J$ or $Y-K$ colours. This is shown by the reddening vector at $z=1.5$ in Fig. 4 . The slope of the reddening vector at other redshifts is very similar. To exclude these objects, we adopt the additional criteria in the following (see Fig. 4):

$(Z-Y)>(Y-K)$

$(Z-Y)>0.5+2.0(Y-J)$

$(Y-J)<1.5$

$(Y-K)<2.0$.

It is less straightforward to exclude T-dwarfs. They are cool $\left(T_{\text {eff }}<1500 \mathrm{~K}\right)$, low-mass stars, and substellar objects that 
have been discovered and studied in these past ten years mainly thanks to the 2MASS (e.g. Burgasser et al. 1999), SDSS (e.g. Leggett et al. 2000), UKIDSS (e.g. Pinfield et al. 2008), and CFBDS surveys (Delorme et al. 2008). Their infrared spectra are dominated by the $\mathrm{CH}_{4}$ and $\mathrm{H}_{2} \mathrm{O}$ absorption bands and by $\mathrm{H}_{2}$ resonant absorption (e.g. Chabrier et al. 2005; Burgasser et al. 2006) that produce a sharp break in their IR colours, which resembles the $Z-Y$ and $Z-K$ colours of high-redshift LBGs. Moreover faint dwarfs can easily be beyond the detection limit even in the $I$ band because of their cold atmospheric temperatures.

Unfortunately, the current uncertainties in cool dwarfs atmospheric models (Helling et al. 2008) do not allow us to compute the expected $Y-J, Y-H$, or $Y-K$ colours with the reliability needed to distinguish T-dwarfs from LBGs. In addition, such distinction is unfeasible on the basis of their morphology/size in our ground-based $Y$ band images, since morphological classification is not reliable at very low $S / N$.

It is, however, possible to estimate their expected number. While cool dwarfs are also found at magnitudes much brighter than those of $z>6$ galaxies, their number density is known to increase towards fainter fluxes (D'Antona et al. 1999; Burgasser 2004), so it is not possible to exclude that some of these objects contaminate our sample of high-redshift candidates. The exact number of expected contaminants depends on the still uncertain parameters constraining the IMF, the spatial distribution of late type dwarfs inside the disk, and the halo of the Galaxy. Observations have excluded these objects being described by a power-law IMF $\Phi(M) \propto M^{-\alpha}$ as steep as the Salpeter one $(\alpha=2.35$ ), with an upper limit at $\alpha=1.5$ (Burgasser 2004). For this reason we considered a worst-case model with an IMF exponent $\alpha=1.5$, and a spatial distribution with height of the Galactic disk $H_{z}=300 \mathrm{pc}$, following the T-dwarfs surface density predictions presented by Burgasser (2004) for a deep survey at high Galactic latitude, we obtained an estimated contamination of $\sim 1.7$ late type dwarfs $\left(T_{\text {eff }}<1750 \mathrm{~K}\right)$ for each Hawk-I pointing. Higher values for the T-dwarfs scale height in the disk (as the $350 \mathrm{pc}$ value proposed by Ryan et al. 2005), or an even steeper IMF, could slightly increase this already pessimistic estimate. However, we underline here that, with so few expected cool dwarfs, the main uncertainties arise from Poissonian fluctuations in their number counts. We note that two cool dwarfs with compatible colours have been indeed found in the GOODS field by Mannucci et al. (2007). They both have $z-Y>1$ in our catalogue: one (ID = 6968 in Mannucci et al. 2007, G1_1713 in our catalogue having $Y=24.45)$ is selected as a candidate drop-out, while the other (ID $=4419$ in Mannucci et al. 2007, G1_5130 in our catalogue, with $Y=23.3$ ) is rejected because it has a significant detection in the $I$ band.

Apart from these well-known classes of possible interlopers, we found that a sample of galaxies selected with the $Z-Y>1$ and $Y-K<1$ criterion is populated also by an unexpectedly large number of faint contaminants showing significant detection in filters covering wavelengths shorter than the redshifted Lyman limit at $z>6$ ( $U$ and $R$ VIMOS; $B, V, I$ ACS).

We show in Fig. 5 the SED and the image in different filters of a typical member of this contaminating population. These objects show a dip in the observed flux in the $Z$ and often in the $I$ band, with a rising continuum in the bluer bands and a large $Z-Y$ colour. Such a spectral energy distribution cannot be reproduced by a straightforward application of the CB07 models. An analysis of the nature of these objects is beyond the scope of the present paper and will be carried out separately. Here, we only note that all of them are relatively faint (mostly $m_{Y}>25$ ).
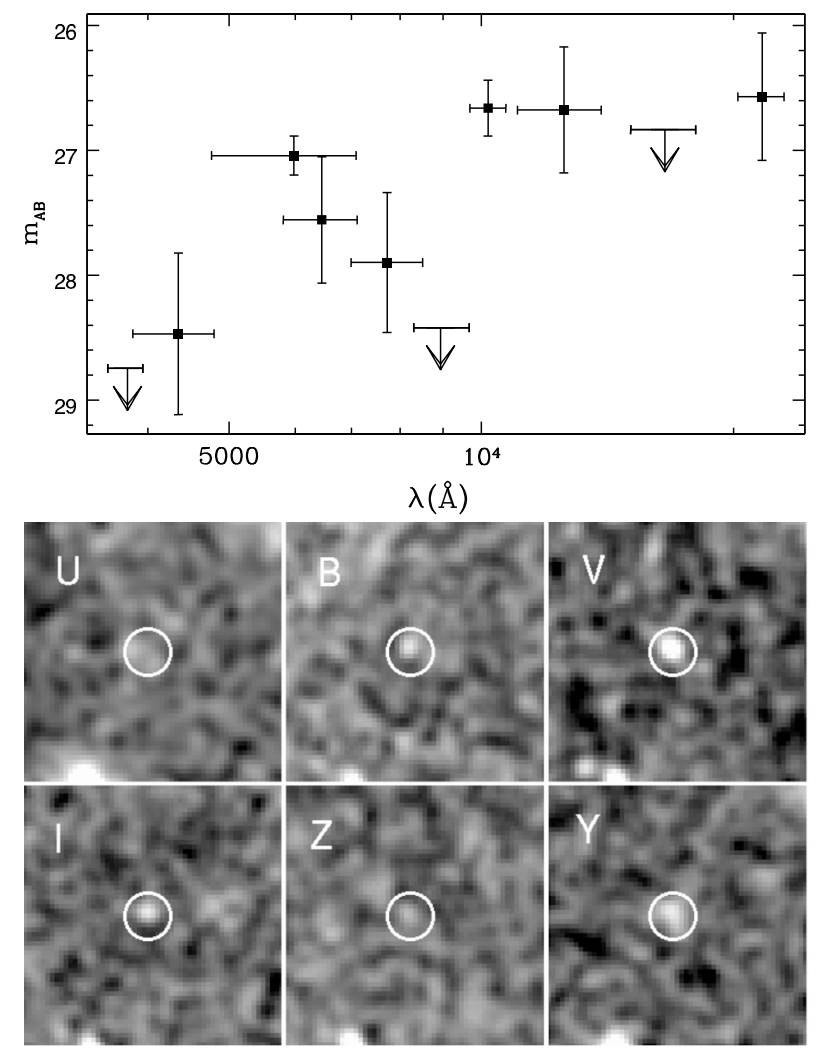

Fig. 5. SED and thumbnail for one of the interlopers with $Z-Y>1$ and detection at shorter wavelengths.

A visual inspection of their ACS images revealed that these objects show a variety of morphologies, at least those detected at good $S / N$. In many cases they are clearly extended, thus indicating their extragalactic nature, although other cases present a point-like morphology. One possibility, especially for those undetected in $J H K$, is that they are faint galaxies with a very blue continuum whose SED is altered by strong emission lines such as in unobscured AGNs, or in star-forming galaxies like the blue compact dwarf galaxies (Izotov et al. 2004, 2007) or the ultra strong emission line galaxies (USELs, Hu et al. 2009).

Although standard prescriptions for the selection of highredshift LBGs already exclude sources detected at short wavelengths, given the rarity of $z>6$ candidates we took particular attention in tailoring reliable criterion to separate our sample of high-redshift candidates from these unexpected lower redshift objects. After looking at the colour distributions of these contaminants and at the distribution of the detected $S / N$ in the $U B V R I$ bands, we decided to adopt a selection criterion that is even more conservative than the one typically adopted by previous surveys. We required the $S / N$ from high- $z$ candidates to be $<2 \sigma_{S / N}$ in all $U B V R I$ bands and $<1 \sigma_{S / N}$ in at least four of them, where $\sigma_{S / N}$ is the estimated rms of the $S / N$ distribution in each band, as described in the next section. In the case of the ACS $B V I$ images we used $S / N$ ratios, and relevant $\sigma_{S / N}$, measured in a smaller aperture (0.6", see Sect. 2.3$)$ to better exploit their higher resolution. To check whether our selection criteria are effective in excluding this population of faint contaminants from the LBG sample, we also analysed the deep ( 230 mag AB at $1 \sigma$ ) public images of the UDF area (Beckwith et al. 2006), which is also covered by our shallower data set (see Fig. 1). Those objects that, on the basis of the conservative criteria discussed above, are individuated as contaminants in our catalogue, 
are all confirmed, at a higher significancy, to have emission in one or more of the blue bands. On the other hand, we effectively select as a high-redshift candidate object UDF-387-1125 already discussed in Bouwens et al. (2004) and recently confirmed in the analysis of the very deep WFC3 images by Bunker et al. (2009), McLure et al. (2010), Oesch et al. (2010), and Yan et al. (2009). Thus we can state that deep optical images such as the ones analysed here $(\sim 29.0$ mag $\mathrm{AB}$ at $1 \sigma)$ are sufficient for separating this class of contaminants from more reliable high-redshift candidates, once conservative selection criteria are adopted. However, we caution that an accurate, dedicated, spectroscopic analysis of both LBGs and contaminants will be necessary for determining the real impact of these objects in LBG searches, since their physical nature is still undetermined.

\section{Simulating the systematic effects}

Although the colour criteria are formally very clear-cut, they are in practice applied to very faint objects, typically close to the limiting depth of the images. At these limits, systematics may significantly affect their detection and the accurate estimate of their large colour terms. To fully evaluate the involved uncertainties we performed extensive imaging simulations, by which we quantified the systematic effects in the object detection, in the measure of their total magnitude as well as in the measure of large colour terms in our images. The output of these simulations will be used in the proper estimate of the LF.

To this purpose, we first used the synthetic libraries described above to produce a large set of simulated galaxies with expected magnitudes in the UBVRIZY filter set in the redshift range $5.5<z<8$. Objects were normalised in the range $Y=$ 24-27.5, following the expected magnitude distribution arising from an LF with index $\alpha=-1.71$, These galaxies were placed at random positions of the GOODS 1 and GOODS $2 Y$-band images, and catalogs were extracted exactly as in the original frames.

As expected, the output of this exercise depends critically on the assumed morphology. In our case, the availability of deep $z$-band ACS images of confirmed $z=5.5-6$ LBGs provides the most natural templates, avoiding further assumptions. Neglecting possible size evolution from $z=6$ to $z=7$, we used the four brightest $z=5.5-6$ LBGs observed with ACS in GOODS, both convolved with the GOODS1/2 PSFs.

To avoid an excessive and unphysical crowding in the simulated images, we included only 200 objects of the same flux and morphology each time, after masking the regions of the images where real objects were detected (corresponding to $6 \%$ of the total area). We repeated the simulation until a total of $10^{5} \mathrm{ob}-$ jects were tested for each field and morphology, for a total of $8 \times 10^{5}$ artificial objects.

These simulations can be used to estimate the impact of different systematics in the various steps of the analysis. First, they can be used to estimate the completeness in the detection procedure. This is shown in the upper panel of Fig. 6, where we plot the fraction of detected versus input objects as a function of the input magnitude. The detection is close to $100 \%$ down to $Y \simeq 26.2$, and fades to $30 \%$ at $Y \simeq 26.7$ (where we find our fainter candidates. We recall that an additional $6 \%$ in the incompleteness stems from the masking done to avoid bright objects.

In addition, they can be used to evaluate the uncertainties in the estimate of the colour criteria that we exploit to detect $z>6.5$ candidates. In particular, the $Z-Y$ colour is a critical feature to identify $z>6.5$ objects. To visualise the effect of noise, we plot in the lower panel of Fig. 6 the conditional probability

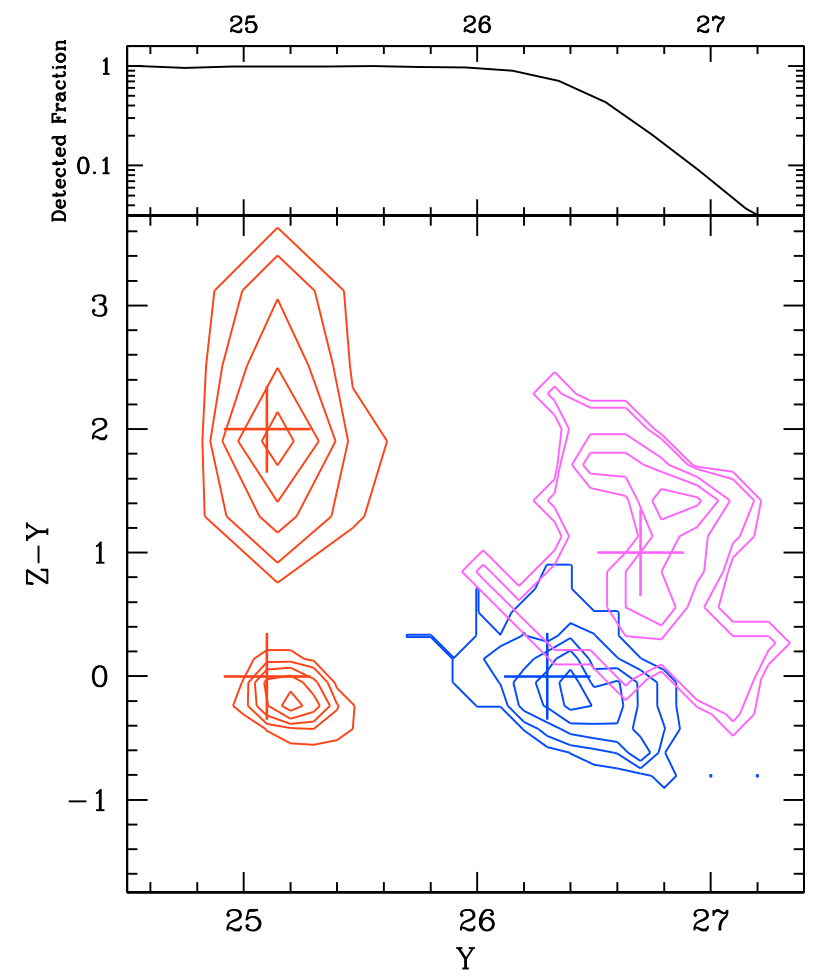

Fig. 6. Upper panel: fraction of detected objects as a function of their input magnitude, as estimated from the simulations described in the text. Lower panel: from the same simulations, contour levels of the conditional probability $P\left(Y_{M},(Z-Y)_{M} \mid Y,(Z-Y)\right)$ that a galaxy with given $Y$ band flux and $Z-Y$ colour is detected with a measured $Y_{M}$ flux and with a measured $(Z-Y)_{M}$ colour. Only four cases are shown, with input values marked by large crosses. Contours are drawn at $0.05,0.15,0.3$, 0.5 , and 0.8 times the peak value of each distribution.

$P\left(Y_{M},(Z-Y)_{M} \mid Y,(Z-Y)\right)$ that a galaxy with given $Y$ band flux and $Z-Y$ colour is detected with a measured $Y_{M}$ flux and with a measured $(Z-Y)_{M}$ colour. Obviously, a fraction of galaxies will not be detected at all in the $Y$ band, as discussed above.

An inspection of Fig. 6 shows the basic feature of the systematics acting on our images. At relatively high $S / N(Y \simeq 25$, $Z \simeq 25$ ), the recovered magnitudes and colours have a narrow scatter, $\sigma_{Y} \simeq \sigma_{Z} \simeq 0.07 \mathrm{mag}$, which increases at lower fluxes $\left(\sigma_{Y} \simeq \sigma_{z} \simeq 0.2\right.$ mag at $Y \simeq 26, Z \simeq 26$ ). At larger $Z-Y$ colours asymmetries becomes evident as the $Z$ magnitude approaches the very detection limit. At the faintest limit, $(Y \simeq 26.8$ and $Z-Y \simeq 1$ ), about $30 \%$ of the objects detected in $Y$ become too faint to be detected in the $Z$ band image.

The simulations in Fig. 6 also show that a 0.15 mag offset exists on average between the input and the recovered magnitude. As discussed above, this comes from the adopted aperture correction, which is computed on unresolved stellar profiles, instead of the LBG profile adopted in the simulations.

Finally, this set of simulations is used to estimate the systematic effects when we use colours at shorter wavelengths, i.e. in the UBVRI bands. In these cases, because of the large IGM and internal $\mathrm{HI}$ absorption, the expected flux in these bands for $z>6.5$ galaxies is far below the detection threshold, or even zero. For this reason a stringent limit on the measured flux in these bands is adopted to remove lower redshift interlopers. However, the $S / N$ estimated by SExtractor may be a poor representation of the actual photometric scatter at low fluxes, due to a combination of factors, such as uncertainties in the estimate of 


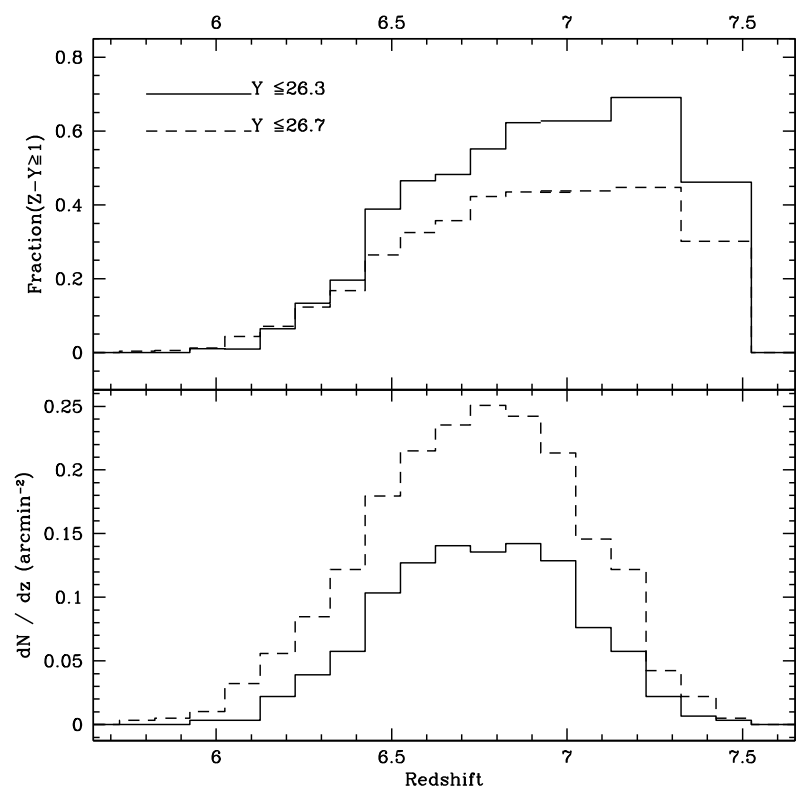

Fig. 7. Upper panel: fraction of $Y$-detected objects passing our criteria $(Z-Y \geq 1$; non-detection blueward of $Z$ ) as a function of redshift, for two limiting magnitudes, $Y=26.3$ (solid line) and $Y=26.7$ (dashed line). Input objects are extracted assuming a constant LF (McLure et al. 2009) from $z=6$. Lower panel: redshift distribution (per unit redshift) for the same sample.

the local background, underestimates of the true rms, or chance superposition of faint blue galaxies along the line of sight.

To account for these effects, we measured the resulting signal-to-noise $\mathrm{SN}$ in the $U B V R I$ images for each simulated objects inserted in the $Y$ one, which should be zero on average. It turns out that the actual distribution of the $S / N$ ratios is wider than the one obtained with SExtractor, which is computed scaling the input weight image. With this set of simulations we thus estimate the "effective" rms $\sigma_{S / N}$, i.e. the rms of the signal-to-noise distribution in each of the 5 images, which is typically about 1.5 . Even taking this wider distribution into account, we also find that the tails of the $S / N$ distribution $(S / N>$ $\left.2 \sigma_{S / N}\right)$ contain more objects than in the case of a pure Gaussian distribution.

As mentioned above, we use the estimated $\sigma_{S / N}$ in all $U B V R I$ bands, requesting that high- $z$ candidates have flux $<2 \sigma_{S / N}$ in all $U B V R I$ bands and $<1 \sigma_{S / N}$ in at least four of them. With our simulation, we estimate that the fraction of true high- $z$ galaxies lost because of this strict criterion is about $\sim 30 \%$. This effect will also be taken into account in our estimate of the LF.

The output of this exercise can be summarised in Fig. 7. The final effect of noise on the $Z-Y>1$ colour is shown in the upper panel, where we plot the fraction of simulated objects detected in $Y$ and with measured $Z-Y \geq 1$ as a function of redshift, for two limiting magnitudes, $Y=26.3$ (solid line) and $Y=26.7$ (dashed line). We remind that, since the objects are extracted from a steep LF, most of them are close to the limiting magnitude. The $Z-Y>1$ colour and the requested non-detection blueward of $Z$ provide an effective cut below $z=6.3-6.4$, which is nearly total at $z<6$, even including the effect of noise. At $z>7$, the fraction of detected objects is less than $100 \%$ because of the combined effects of incompleteness in the detection and of the requested non-detection blueward of $Z$, which rejects some genuine high- $z$ galaxies because of photometric scatter.

The most important output is shown in the lower panel of Fig. 7, where we plot the expected redshift distribution of our sample, as extracted from the simulations. In this case, counts are normalised assuming that the LF remains constant from $z=6$ with the values provided by McLure et al. (2009). Since we populate the input catalogue following a LF with a realistic slope, we expect that this redshift distribution is a good estimate of the redshift selection function of our survey. The low-redshift cut-off comes from the $Z-Y>1$ colour and the requested non-detection blueward of $Z$, as discussed above. At higher redshift, the cutoff is caused by the decreasing number of expected galaxies in a magnitude-selected sample - as shown in Fig. 3, objects of given $L$ become rapidly fainter at $z>7.3$. In this case, the highredshift tails of the distribution is sensitive to the evolution of the LF: in case of a marked drop in luminous objects at high redshift, with respect to the McLure et al. (2009) LF, the distribution will be skewed toward the lower redshift boundary. This plot clearly shows the basic feature of our $Y$-band selected approach, which allows sampling of the LBG LF in a well-defined narrow redshift range. This redshift range is narrower than the corresponding one in $z-J$ selected samples.

\section{Detected $z>6.5$ galaxies}

Based on the results of the previous sections, we finally selected the $z \geq 6.5$ candidates by adopting the following criteria:

1) we included only objects detected in the deepest regions of the images (rms not greater than $1.5 \times$ its minimum value) with total magnitude $Y \leq 26.8$;

2) we require:

$$
\begin{aligned}
& (Z-Y)>1.0 \\
& (Z-Y)>(Y-K) \\
& (Z-Y)>0.5+2.0(Y-J) \\
& (Y-J)<1.5 \\
& (Y-K)<2.0
\end{aligned}
$$

3) we require that the $S / N$ (as measured in units of the simulation-calibrated $\left.\sigma_{S / N}\right)$ is less than 2 in all $U B V R I$ bands, and above 1 in one of these bands only. Figure 8 shows the position of all objects of our sample in the $Y-J$ vs. $Z-Y$ plane, with the final candidates and the two known brown dwarfs shown highlighted.

In practice, the limit on the $Y-J<1.5$ and $Y-K \leq 2$ colours are ineffective. Indeed, all the objects with $Z-Y \geq 1$ are very blue in the near-IR, having $Y-J<1.2$ and $Y-K<1.7$, and $90 \%$ of them have $Y-J<0.5$ and $Y-K<1$. As a result, the critical requirement to select "bona-fide" high- $z$ candidates is the non-detection in all the bands blueward of the $Z$ filter. In our catalogue we detect 139 objects with $Z-Y \geq 1$ and $Y \leq 26.8$, only 9 of which pass the additional criteria on the non-detection in the $U B V R I$ bands.

Most of our candidates are marginally detected at very low $S / N \simeq 2$ in the $Z$ and/or $J$ images, which makes it mandatory to critically assess their reality. The first and most obvious check to be done is with the available NICMOS F110W and F160W images in the GOODS area. Unfortunately, these images do not cover the whole Hawk-I pointings with the required depth. Out of 4 of our candidates falling on suitably deep images, we clearly detect 3 of them with $F 110 \mathrm{~W}$ and F160W magnitudes consistent with our $Y$ one. The fourth one is undetected in the $F 160 \mathrm{~W}$ with $m_{F 160 W}-m_{Y}>1$ ( $F 110 \mathrm{~W}$ is not available). As a consequence we have removed this object from our sample. 
M. Castellano et al.: Evidence of a fast evolution of the UV LF at $z>6$

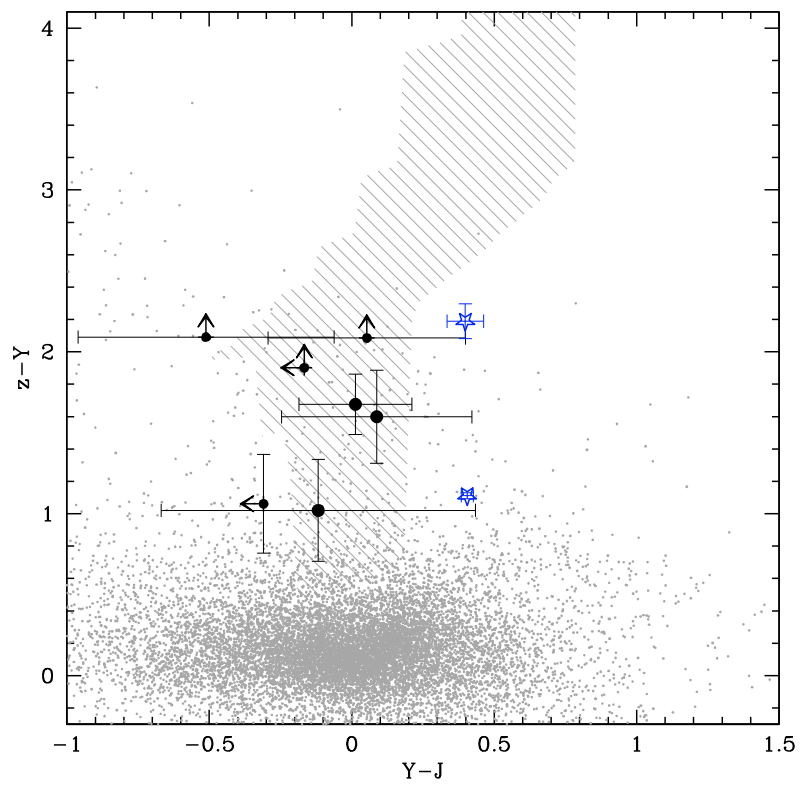

Fig. 8. Position of the high- $z$ candidates in the $Z-Y$ vs. $Y-J$ colour plane (black large dots). Upper limits are computed at the $1 \sigma$ level. The shaded area shows the location of the expected colours of CB07 models, at $z \geq 6.5$. The faint gray dots show the rest of the sample. Objects fulfilling the selection criterion are rejected because of their detection in at least one of the UBVRI images (see text for details). The two Brown Dwarfs already identified by Mannucci et al. are shown as stars. The one in the upper right is selected as a high- $z$ candidate on the basis of its $U B V R I Z Y$ photometry, but removed from the final sample.

A similar check can be done on the IRAC images. Taking the actual depth of the IRAC images into account, a detection is not definitely required to confirm the existence of these candidates. Indeed, using the same synthetic libraries described above, we expect colours $m_{Y}-m_{\mathrm{IRAC}}$ in the range $-2 /+2$ mag, primarily depending on the age, mass, and dust content of the galaxies. Since the detection limit in IRAC does not exceed 26.5, grossly the same AB limit of our candidates, we can expect that only a fraction of these galaxies are detected in the IRAC images. Indeed, 3 (out of 8 ) objects are clearly detected in the IRAC $3.6 \mu$ channel (IRAC 36 hereafter): G1_1921, G2_1713, and the object G1_2631 already detected in NICMOS. Further three objects (G2_1408, G2_2370, and G2_6173) are marginally detected due to blending with nearby sources. Two of the IRAC detected objects are the faintest ones in our sample (G1_1921 and G1_2631), so the IRAC detection confirms their reality.

We computed aperture photometry (corrected to total) on both the $3.6 \mu$ and the $4.5 \mu$ IRAC images. None of our IRAC detected objects shows a (3.6-4.5) index over $\sim 0.4$, in agreement with the colours expected for high-redshift LBGs. On the other hand, previous studies have found that cool dwarf stars show a broad range in the $4.5 \mu$ luminosity, yielding values in the 3.6-4.5 colour index that can be much larger than those of our objects (up to $2.0 \mathrm{mag}$, see Patten et al. 2006; Helling et al. 2008), although lower colour indexes have also been observed for these objects.

As already described in Sect. 3.2, one of our candidates was already detected as a potential high- $z$ candidate by Mannucci et al. (2007) and associated to a Galactic brown dwarf. We show in Fig. 8 the position of this and of the other Galactic brown dwarf found by Mannucci et al. (2007), as well as of the 7 other candidates. It is interesting to note that, given the high $S / N$ that we obtain on these relatively bright brown dwarfs, the measured
Table 1. Hawk-I z-drop candidates in Goods-S.

\begin{tabular}{ccccc}
\hline \hline ID & RA (deg) & Dec (deg) & $Y$ & $Z-Y$ \\
\hline G1_149 & 53.157038 & -27.930707 & 26.06 & 1.60 \\
G1_1921 & 53.19538 & -27.835421 & 26.71 & 1.02 \\
G1_2631 & 53.106022 & -27.848123 & 26.64 & 1.06 \\
G2_1408 & 53.177382 & -27.782416 & 26.37 & $>2.1$ \\
G2_2370 & 53.094421 & -27.716847 & 25.56 & 1.68 \\
G2_4034 & 53.150019 & -27.744914 & 26.35 & $>2.1$ \\
G2_6173 & 53.123074 & -27.701256 & 26.53 & $>1.9$ \\
\hline
\end{tabular}

$Y-J$ colour is larger than that expected for an LBG at $z>$ 6.5. This analysis further supports the conclusions of Mannucci et al. (2007), therefore we remove G1_1713 from our sample of "bona-fide" high- $z$ candidates.

In the attempt to identify further brown dwarfs, we also investigated the morphology of the candidates in the NICMOS images, where available. Object G2_2370 has a full width of about $0.45^{\prime \prime}$, close to the 0.4 value in NICMOS, but with a nonnull ellipticity 0.3 . Object G2_1408 is clearly resolved, with $F W H M \simeq 0.65$. Also considering that several LBGs with spectroscopic redshifts in GOODS have a NICMOS PSF consistent with the stellar one, we keep both of them in our sample.

After removing the Mannucci et al. (2007) brown dwarf and the object undetected in deep NICMOS pointings from our sample, we are left with 7 candidates: their position and properties are given in Table 1. The relevant thumbnails are given in Fig. 9.

We performed a stacking of all the thumbnails in the $U B V R I Z Y J H K$ images. We confirm that objects are undetected in the $U B V R I$ images, are detected in the $J$ band average image, and exhibit an average colour $Z-Y \simeq 2$. The resulting SED provides an excellent photometric redshift at $z=6.8$. Relevant thumbnails and SED are shown in Fig. 10.

We checked whether any of the previously published, NICMOS detected, $z>6$ candidates in the UDF area (Bouwens et al. 2004; Bouwens \& Illingworth 2006; Oesch et al. 2009) are present within our sample. We find that our candidate G2_1408 is the object UDF-387-1125 in Bouwens et al. (2004). All the other objects are not detected with our extraction parameters. We notice, however, that most of these objects have faint magnitudes in the J110 and H160 NICMOS bands ( 26.8-27.0 AB), while we adopted conservative selection criteria in order to have a low number of spurious detections at the faintest magnitudes in our sample ( $Y=26.8$, see Sect. 2.2), so it is not surprising that we are missing objects detected in the very deep NICMOS pointings over the UDF. The colours of all the selected objects are consistent with low values of $Y-J$ (or $Y-K$ ) plane, as shown in Fig. 8. Since higher values of $Y-J$ (even within our threshold $Y-J \leq 1.5)$ are typical of galaxies at $z>7.2$, this confirms our expectations that the redshift distribution is in practice limited at this value. We also checked other recent results on the GOODS area presented by Hickey et al. (2009) and by Wilkins et al. (2009).

Hickey et al. (2009) exploit part of the same Hawk-I dataset presented here but reach a shallower magnitude limit $(Y \sim$ 25.7-25.9). Their candidate ID 9697 is our candidate G2_2370, while their object ID 9136 is rejected in our sample because of $S / N$ in the $I$ band slightly above our threshold. Their two other candidates show significant detection in the bluer bands and so are probably lower redshift interlopers as discussed also by the authors.

Wilkins et al. (2009) present an analysis of the WFC3 ERS data covering the northern portion of our "GOODS2" field. 

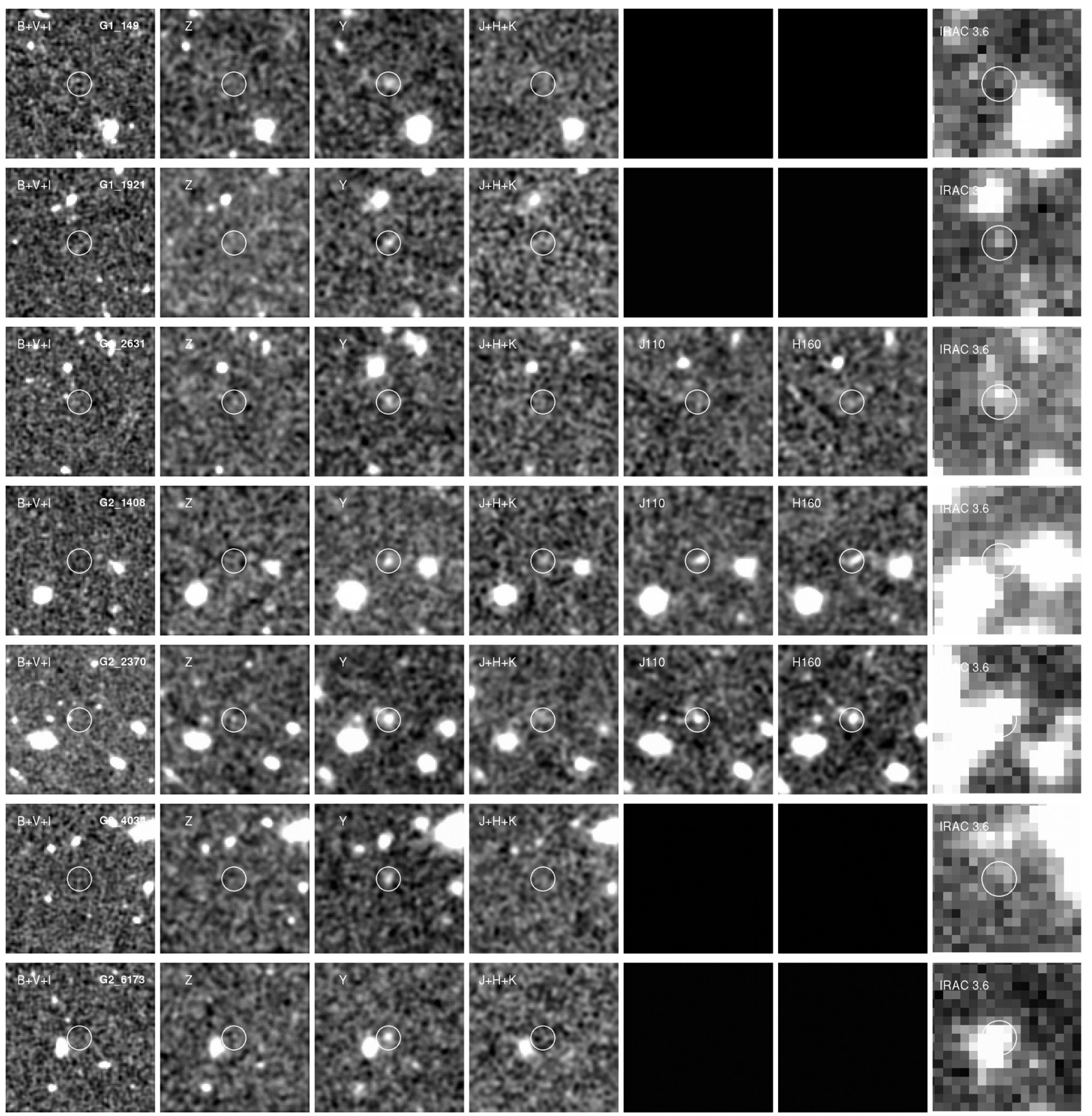

Fig. 9. Thumbnails showing the images of the 7 selected high-redshift candidates in the different observed bands.

Their candidate \#4 is our object G2_6173, although they measure a fainter $Y$ magnitude. Of their two candidates having $Y<26.8$ we reject object \#1 because of non negligible $S / N$ in the $I$ band, while object \#2 is too close to a bright $(Y \sim 20)$ source to be effectively de-blended in our ground-based images and it is not present in our catalogue.

We note that we estimated that a $30 \%$ of real sources are missed with the strict rejection criteria in the blue bands adopted in the present analysis. This is consistent with object ID 9136 in Hickey et al. (2009) and object \#1 in Wilkins et al. (2009) being real high-redshift galaxies whose high $I$-band $S / N$ in our catalogue comes from photometric scatter, although their non negligible $S / N$ in the $I$ band might be due to a redshift near the lower limit of the redshift selection window. Potential missed detections because of photometric scatter, or because of objects falling near other bright sources, are fully taken into account in the LF estimate presented in the next section based on extensive imaging simulations.

We finally checked whether there is any X-ray emission detectable in the deep X ray images (Luo et al. 2008). No source was individually detected. In the stacked image, the $90 \%$ count rate $(0.5-2 \mathrm{keV})$ limit is $2.41 \times 10^{-6}$ counts/s, for a total exposure time of $1.2 \times 10^{7} \mathrm{~s}$. The resulting luminosity is $\leq 7.8 \times$ $10^{42} \mathrm{erg} / \mathrm{s}$ in the band from 2 to $10 \mathrm{keV}$ (assuming a power-law 

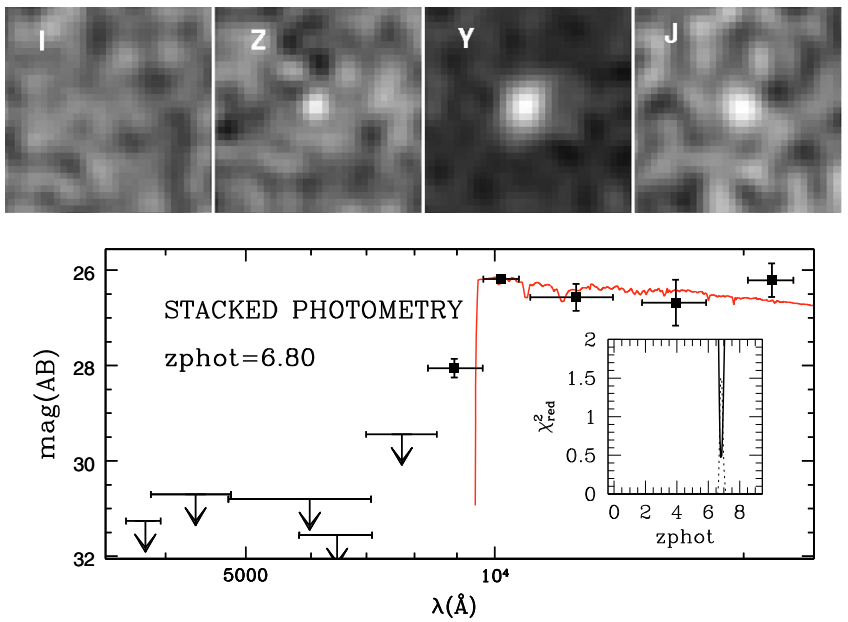

Fig. 10. Upper: thumbnails showing the stacked images of the 7 selected high-redshift candidates in the observed bands shown in the legends. Lower: resulting SED, with relevant photometric redshift at $z=6.8$.

spectrum with a energy index -0.4$)$. Assuming a bolometric correction factor of 20 and an Eddington-limited accretion rate, we derive a limit on the black hole mass of about $\leq 10^{6} M_{\odot}$.

\section{The evolution of the LF}

To estimate from our detections the most likely LF we used an approach that fully accounts for the expected systematics in the detection process, as done by, e.g., Bouwens et al. (2007), Mannucci et al. (2007) and McLure et al. (2009). We refer in the following to the luminosity $L$ as measured at $1500 \AA$ rest frame.

First, we assume that the LF can be described by the usual Schechter function with parameters $\phi, \alpha$, and $M_{*}$ (Schechter 1976). Unfortunately, we are unable to constrain the slope of the LF, since our faint limit is close to the expected value of the characteristic luminosity $M_{*}$. For this reason, our results are grossly insensitive to the value of the slope index $\alpha$, which we fix to the value $\alpha=-1.71$ of the $z \sim 6$ LF by McLure et al. (2009), close to $\alpha=-1.74$, used by Bouwens et al. (2007). We explicitly tested this assumption by fixing $\alpha$ to significantly different values $(\alpha=-1.4,-2.0)$, without finding significant differences.

We assume that the other two parameters evolve in redshift from their value at lower $z=z_{0}$ value:

$\log (\phi(z))=\log \left(\phi\left(z_{0}\right)\right)+\mathrm{d} \log (\phi) / \mathrm{d} z \cdot\left(z-z_{0}\right)$

$M_{*}(z)=M_{*}\left(z_{0}\right)+M_{*}^{\prime} \cdot\left(z-z_{0}\right)$.

We note that our parametrisation is analogous to the one adopted by Bouwens et al. (2008). While Bouwens et al. (2008) used this parametrisation to fit their values at different redshifts (in the range $4<z<10$ ), we explicitly vary the Schechter parameters within our redshift range $6.4<z<7.2$, which might be important in case of a strong evolution. We also tested a purely linear evolution of $\phi$, which turned out to produce consistent results.

In principle, all four parameters could be left free in the minimisation process. However, given the size and depth of our sample, we assume for $M_{*}\left(z_{0}\right)$ and $\phi\left(z_{0}\right)$ the observed values at slighter lower redshifts $z \simeq 6$ and evaluate the evolutionary terms $M_{*}^{\prime}$ and $\mathrm{d} \log (\phi) / \mathrm{d} z$ alone.

During the minimisation process, for any given value of the free parameters, we Monte Carlo extract a sample of objects with redshift $z, 1500 \AA$ A rest-frame absolute magnitude $M$, randomly
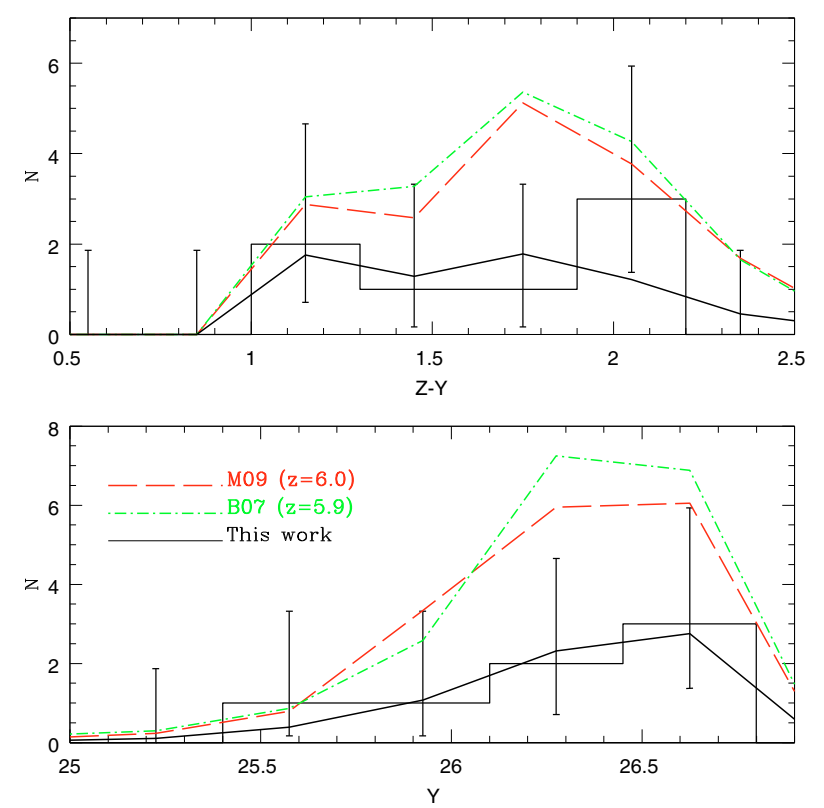

Fig. 11. $Y$ and $Z-Y$ distributions of the high redshift candidates and of our best-fit evolving LF at $z>6$ (black solid line), compared with the expected values from recent estimates of the LF at $z \sim 6$ by Bouwens et al. (2007) (B07, green dot-dashed line) and McLure et al. (2009) (M09, red dashed line), as computed according to the simulations described in Sect. 6.

adding different $E(B-V)$ and metallicities as obtained by the CB07 models shown in Figs. 3 and 2. We include Ly $\alpha$ emission with a Gaussian distribution with standard deviation $30 \AA$. All these galaxies are extracted from the (larger) simulations described in Sect. 4. This way, we can convert their rest-frame $M_{\mathrm{UV}}$ into observer-frame magnitudes in the same bands as are used in our observed sample, taking into account all the uncertainties involved in the observations discussed in Sect. 4: detection completeness, photometric scatter and random fluctuations in the $S / N$ measure due to overlapping interlopers or other effects.

The distributions of magnitudes and colours for each Monte Carlo simulation are scaled to the observed area in the GOODS-S field (after excluding the fraction of area lost because of the presence of lower redshift objects) and compared to the observed ones with a maximum likelihood test under the assumption of simple Poissonian statistics.

To provide a visual example of the significance of the results, we show the expected number counts for three cases of the evolution of the LF in Fig. 11, comparing them with our observed counts. It is immediately appreciated that, barring for the moment large fluctuations due to cosmic variance, the number counts of our candidates imply a strong evolution of the LF in the relatively short cosmic time elapsed from $z \simeq 6$ to $z=6.8$.

To constrain the evolution of the LF, for each simulated population, and for each of the two distributions, we build the likelihood function $\mathcal{L}$

$\mathcal{L}=\prod_{i} \mathrm{e}^{-N_{\mathrm{exp}, i}} \frac{\left(N_{\mathrm{exp}, i}\right)^{N_{\mathrm{obs}, i}}}{\left(N_{\mathrm{obs}, i}\right) !}$

where $N_{\mathrm{obs}, i}$ is the observed number of sources in the magnitude (colour) interval $i, N_{\exp , i}$ is the expected number of sources in the same magnitude (colour) interval, and $\Pi_{i}$ is the product symbol. The final likelihood assigned to each given model is the product of the likelihoods computed for the magnitude and colour 


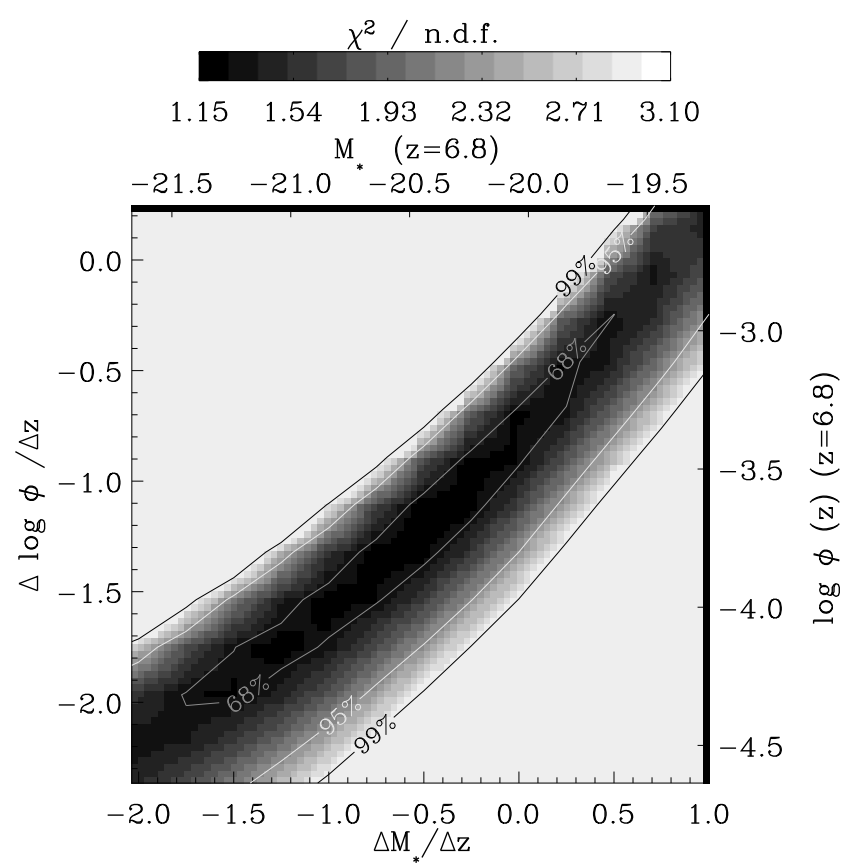

Fig. 12. $\chi^{2}$ contour levels for the $\mathrm{d} \log (\phi) / \mathrm{d} z, M_{*}^{\prime}$ parameters derived for the Schechter-like LF. The lower and left axis refer to the evolutionary terms $M_{*}^{\prime}$ and $\mathrm{d} \log (\phi) / \mathrm{d} z$ (see text for details), the upper and right axis refer to the $M_{*}$ and $\phi$ values at the median redshift estimated for our sample $(z=6.8)$.

distributions separately. We iterate this procedure for a grid of $\left(\mathrm{d} \log (\phi) / \mathrm{d} z, M_{*}^{\prime}\right)$ values.

The colour plot in Fig. 12 shows the 68\%, 95\%, and 99\% likelihood intervals on the evolutionary terms $M_{*}^{\prime}$ and $\mathrm{d} \log (\phi) / \mathrm{d} z$ (left and bottom axes) and for the resulting Schechter parameters at the median redshift $z=6.8$ of our sample (top and right axes). In the same plot, the colour code refers to the $\chi^{2}$ distribution obtained under the usual assumption $\chi^{2}=-2.0 \cdot \ln (\mathcal{L})$. It is evident that the absence of any evolution in both parameters $\left(\mathrm{d} \log (\phi) / \mathrm{d} z=0\right.$ and $\left.M_{*}^{\prime}=\mathrm{d} M_{*} / \mathrm{d} z=0\right)$ with respect to the best-fit values at $z=6$ is ruled out at $\gtrsim 99 \%$ confidence level. This is shown more clearly by Fig. 13 where our $68 \%$ and $95 \%$ likelihood intervals at $z=6.8$ are displayed with the likelihood contours computed in a self-consistent way by Bouwens et al. (2007) at redshifts 3.8, 5.0, 5.9, and 7.4.

The formal maximum of the maximum likelihood lays at $\mathrm{d} \log (\phi) / \mathrm{d} z=-0.89 \pm 0.21$ and $M^{\prime}=\mathrm{d} M_{*} / \mathrm{d} z=-0.25 \pm$ 0.56 (errors include the effect of cosmic variance, see below). At face value, our best-fit model indicates mostly a decrease in the normalisation factor $\phi$ when compared to the best-fit parameters at $z \sim 6$ (Fig. 13). However, the relatively few galaxies that we have in our fields do not allow us to put tight constraints on the exact combination of Schechter parameters: as shown by the elongated shape of the contour levels in Fig. 13 the two parameters are highly degenerate. We indeed find that the position of the maximum is poorly constrained, and somewhat dependent on the details of the simulations, like step or number of simulated galaxies. We therefore only provide its value for reference, and focus on the overall range of allowed values at the $1 \sigma$ contour or on integrated quantities.

The effects of cosmic variance are a significant concern in our case, since all our data come from a single pointing that includes most of the previous NICMOS-based surveys. Indeed, cosmic variance effects are not independent of those potentially

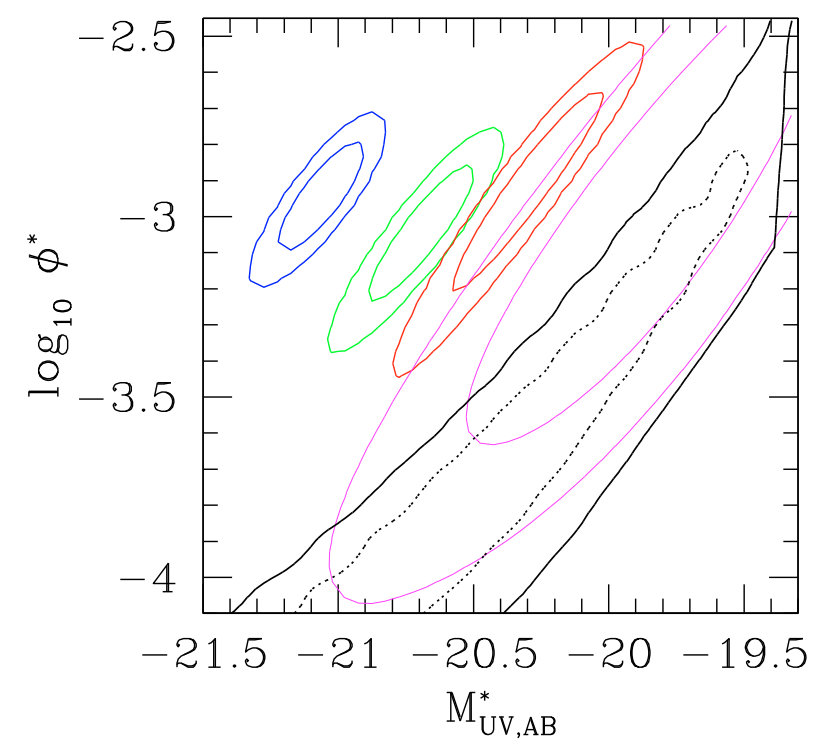

Fig. 13. 68\% (dotted line) and 95\% (continuous line) $\chi^{2}$ contour levels for the value of $M_{*}$ and $\phi$ at $z=6.8$ as estimated from our sample of $Y$-detected LBGs. Blue, green, red and magenta contours are the $68 \%$ and $95 \%$ likelihood intervals on the Schechter parameters as estimated by Bouwens et al. (2007) at redshifts 3.8, 5.0, 5.9, and 7.4, respectively.

affecting the Bouwens et al. (2008) results. The cleanest way to get rid of these effects is to analyse of several independent fields when applying the same selection criteria. For this reason, our survey continues by covering two completely disjointed fields with the same setup. For the moment, we can evaluate the possible impact of cosmic variance by measuring the relative variance within 200 samples bootstrapped from the Millennium Simulation presented by Kitzbichler \& White (2007), which is considered to accurately reproduce clustering properties even at very high redshift (e.g. Overzier et al. 2009). We use an area as large as our Hawk-I data set and we apply a corresponding photometric selection criteria on galaxies at $6.4<z<7.2$, without any constraint on the distribution of host haloes. We estimate that a cosmic variance of $\sim 30 \%$ affects the number counts of z-drop LBGs in our $\sim 90 \mathrm{arcmin}^{2}$ area. We estimated the resulting effect on the $\phi$ and $M_{*}$ parameters changing by $\pm 30 \%$ the observed number counts, and finding the relevant best-fit parameters. These offsets were added in quadrature to the Hessian errors computed around the best fit value.

In this way we obtain a best-fit interval for the evolutionary terms along with their associated uncertainty, which includes both Poisson and cosmic variance noise: $\mathrm{d} \log (\phi) / \mathrm{d} z=-0.89 \pm$ 0.21 and $M^{\prime}=\mathrm{d} M_{*} / \mathrm{d} z=-0.25 \pm 0.56$, resulting in $\Phi^{*}=$ $0.35_{-0.11}^{+0.16} \times 10^{-3} \mathrm{Mpc}^{-3}$ and $M^{*}(1500 \AA)=-20.24 \pm 0.45$ at $z=6.8$.

Fortunately, the degeneracy in the $M_{*}$ and $\phi$ best-fit values is not reflected in a comparable uncertainty in the number density of bright galaxies, i.e. in the integral of the LF up to $M_{*}$. Indeed, because of the correlation between the acceptable values of $M_{*}$ and $\phi$, an increase in the normalisation $(\phi)$ is compensated for by a decrease in the characteristic luminosity, such that the integral up to $M \simeq M_{*}$ remains grossly constant. By integrating the best-fit UV LF up to $M=-19.0$ we obtain an UV luminosity density $\rho_{\mathrm{UV}}=1.5_{-0.9}^{+2.0} \times 10^{25} \mathrm{erg} \mathrm{s}^{-1} \mathrm{~Hz}^{-1} \mathrm{Mpc}^{-3}$. The errors are computed by integrating all the UV LF that are acceptable at the $68 \%$ level.

For comparison, the integral of the $z=6 \mathrm{UV}$ LF of McLure et al. (2009) up to the same magnitude limit yields a 
Table 2. Stepwise determination of the UV LF at $z \sim 6.8$.

\begin{tabular}{cc}
\hline \hline$M_{1500}$ & $\phi\left(10^{-4} \mathrm{Mpc}^{-3} \mathrm{mag}^{-1}\right)$ \\
\hline-22.0 & $0.02_{-0.02}^{+0.33}$ \\
-21.0 & $0.19_{-0.19}^{+0.47}$ \\
-20.0 & $2.20_{-1.47}^{+1.47}$ \\
\hline
\end{tabular}

$\rho_{\mathrm{UV}}=5.6_{-2.3}^{+3.1} \times 10^{25} \mathrm{erg} \mathrm{s}^{-1}$. Our estimate thus implies a drop of a factor $\sim 3.5$ in the UV luminosity density from $z=6$ to $z=6.8$. We can convert this value in a star formation rate density following the standard formula by Madau et al. (1998) and applying the extinction correction of Meurer et al. (1999) (considering an average UV slope $\beta=-2.0$ ). We obtain $S F R D=2.8_{-1.6}^{+2.7} \times$ $10^{-3} M_{\text {sun }} \mathrm{yr}^{-1} \mathrm{Mpc}^{-3}$.

To provide a more straightforward comparison to other results in the literature, we also computed the LF for our $z$-dropout sample in a stepwise form (see, e.g. Bouwens et al. 2008, for a discussion of this method). Briefly, the stepwise method assumes that the rest-frame LF of galaxies can be approximated by a binned distribution, where the number density $\phi_{i}$ in each bin is a free parameter. This non-parametric approach allows us to constrain the number density of galaxies at different magnitudes without assuming a Schechter-like shape.

We have assumed that the LF is made of three bins in the interval $-22.5<M_{1500}<-19.5$, corresponding to the range sampled by our observations. We also assume that galaxies are uniformly distributed within the bins, with number densities $\phi_{i}$ to be determined. We exploit the same set of simulations described in Sect. 4 to compute the distribution of observed magnitudes originating in each bin, scaled to the observed area in the GOODS-S field. We then find the combination of $\phi_{i}$ that best reproduces the magnitude distribution of our observed objects with a simple $\chi^{2}$ minimisation. The results are reported in Table 2 and are displayed in Fig. 14 along with recent results from the literature and our best-fit Schechter LF discussed above.

Our two independent determinations of the LF at $z \sim 6.8$ (stepwise and maximum likelihood) are in perfect agreement. The agreement at the bright end with the densities estimated by Bouwens et al. (2008) and Ouchi et al. (2009) is remarkable, considering the widely different data sets and selection techniques used and the large uncertainties given by the low number of objects in our sample. The disagreement at the faint end between our best-fit LF and the points by Oesch et al. (2010) is probably the result of the fixed slope $\alpha=-1.71$ we had to assume for our maximum likelihood test, as discussed above, and it is consistent with a steepening of the faint end, as suggested by the authors.

\section{Predictions for future surveys}

It is interesting to use the values of the Schechter parameters found in the previous section to estimate the number of detections that may be obtained with future surveys. Clearly, the large errors in our determination make this exercise uncertain, but it is nevertheless useful to design future surveys from ground and space.

From the ground, the relative efficiency of the $Y$ band allows us to cover significantly large areas of the sky. This is useful both to beat cosmic variance and to identify potential spectroscopic targets. Using our best-fit values of $\mathrm{d} \log (\phi) / \mathrm{d} z, L_{*}^{\prime}$ (and considering the two values $\alpha=-1.7$ and $\alpha=-1.9$ ) in Fig. 15 we show the combination of area and limiting magnitude needed to

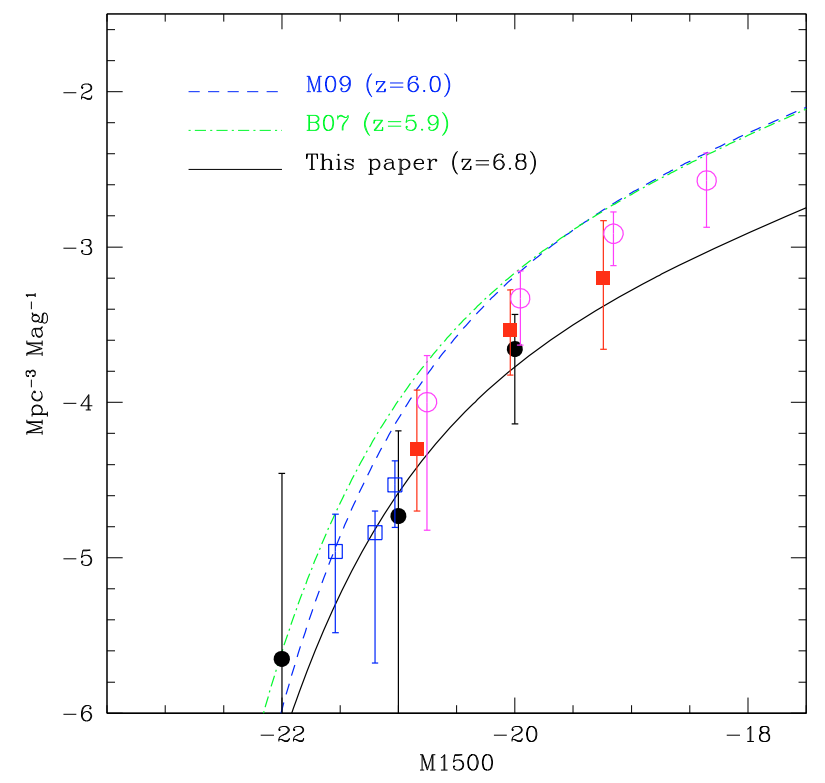

Fig. 14. Number densities in three rest-frame magnitude intervals estimated for our Hawk-I data set in a stepwise form (black circles and error bars), along with the results by Bouwens et al. (2008) for NICMOS detected objects (red filled squares), Ouchi et al. (2009) (SUBARU, blue empty squares) and Oesch et al. (2010) (WFC3, magenta empty circles). The black solid line is our best-fit LF discussed in the text. For a comparison we show the recent determinations of the LF at $z \sim 6$ by Bouwens et al. (2007) (B07, green dot-dashed line) and McLure et al. (2009) (M09, blue dashed line).

detect a given number of galaxies at $6.4<z<7.2$. We also show the position of our present data (labelled as Hawk-I GOODS), of the planned Ultra-VISTA observations of the COSMOS field, of the WFC3 pointing over the UDF (Oesch et al. 2010), and of a $\sim 20 \mathrm{~h}$ pointing with the $115 \mathrm{~W}$ filter of JWST-NIRCam. For the Ultra-VISTA COSMOS survey, we consider the case of the deepest non-contiguous observations for a total of $0.73 \mathrm{deg}^{2}$. We stress that these predictions do not include the fraction of galaxies lost because of different effects. In our case, the major sources of losses are the incompleteness in the photometric detection and the scatter (intrinsic and observational) in the $Z-Y$ colour that we use as threshold, leading to a loss of about $30 \%$ of the candidates. For this reason, the number of candidates that we detect is about half of the expected numbers in Fig. 15. Clearly, surveys with similar incompleteness levels should expect a comparable reduction of the observed numbers. The regression of the bright side of the LF, combined with its exponential slope, results in a flattening of the expected cumulative numbers at bright fluxes even in wide areas. In practice, it is very difficult to detect galaxies brighter than 25 mag even over areas of about $1 \mathrm{deg}^{2}$, such that the detection of galaxies brighter than 24.5 in Ultra-VISTA would be a formal violation of our LF.

The two straight red lines in Fig. 15 show the expected position in the area-limiting magnitude plane of the two groundbased surveys (Hawk-I GOODS and Ultra-Vista COSMOS) if they were conducted over more pointings with the same total exposure time ( $32 \mathrm{~h}$ and $150 \mathrm{~h}$, respectively). By increasing the number of pointings the total number of candidates decreases, with a loss of statistical robustness. For a relatively small number of pointings, this is likely counterbalanced by the decrease in the scatter due to cosmic variance. In essence, for a given instrument and total investment of time, the highest return in a statistical sense is given by a modest number of independent pointings. 


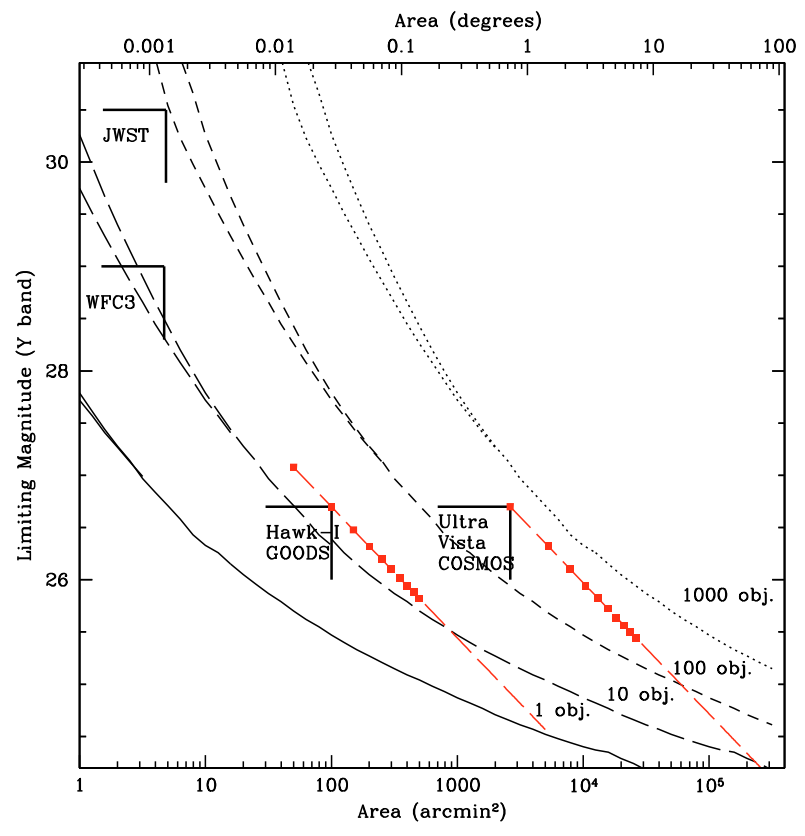

Fig. 15. Expected number of $6.4<z<7.2$ galaxies as a function of area and limiting magnitude for present and future ground-based surveys and for deep pointings with HST-WFC3 and JWST-NIRCam (see text for details). Black curves show the combination of area and limiting magnitudes necessary to collect $1,10,100$ or 1000 galaxies in this redshift range. Estimates are based on our best-fit values of $\mathrm{d} \log (\phi) / \mathrm{d} z, M_{*}^{\prime}$ and considering the two values $\alpha=-1.7$ and $\alpha=-1.9$ (upper and lower curves, respectively). Note that incompleteness and noise effects are not included in the computation and could significantly decrease the number of candidates eventually detected. The red straight lines show the position of the two surveys if they were conducted over more pointings with the same total exposure time. Each dot represent an additional pointing.

Only WFC3 on HST has the required IR sensitivity to detect galaxies at even higher redshifts, ahead of the advent of JWST. For this reason, several surveys are starting or are being planned using the WFC3 IR filters. In Fig. 16 we provide the predicted cumulative number counts for galaxies in different redshift ranges, selected using either $F 105 M-F 125 W \geq 1$ (that corresponds to the redshift range $z=7.8-9$ ) or $F 110 \mathrm{~W}-F 160 \mathrm{~W} \geq$ 1 ( $z \geq 8.8)$. In both panels, the magnitudes in the $F 125 W$ and F160W filters are computed from $L_{1500}$ using the same CB07 described above. We provide the number counts expected in the case of a non-evolving LF from $z=6$ (clearly an upper limit), the predictions from the evolving LF of Bouwens et al. (2008) (which was drawn from $z=4$ to $z=10$ ), and the extension to $z=10$ of our LF. For the extrapolation of our LF, we also show the whole region predicted by extending from $z=7$ to $z=10$ all the LFs acceptable at the $1 \sigma$ level found from our analysis at $z=7$.

Apart from the unrealistic case of a constant LF beyond $z=6$, the number density of LBGs at $z \geq 8$ is expected to be very low and requires a combination of large areas and extreme depth to collect a sizeable sample. Taking the WFC3 size into account (about $4.6 \operatorname{arcmin}^{2}$ ), it is necessary to reach continuum magnitudes at least $m_{F 125 W} \simeq m_{F 160 W} \simeq 28$ to detect at least one dropout in a single WFC3 pointing.

Figure 16 also shows that the evolution of the LF resulting from this work is faster than the Bouwens et al. (2008) one. Assuming that this evolution continues at the same rate at higher redshift, there would be less expected dropouts at higher

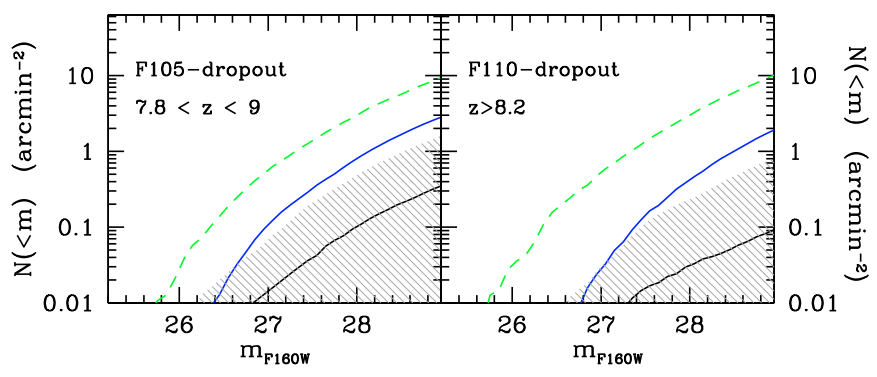

Fig. 16. Expected cumulative number counts of high redshift galaxies as a function of the $F 160 \mathrm{~W}$ magnitude, for different redshift ranges and different choices of the evolutionary LF. The adopted colour cuts and the corresponding redshift ranges are shown in the legend. In both panels. the green dashed line is the prediction from a constant (from $z=6$ to $z=10$ ) UV LF. The black solid line is our best fit evolving LF, while the shaded area shows the predicted number counts for all the LF parameters acceptable at the $1 \sigma$ level in our survey. The blue continuous line is the evolving LF from Bouwens et al. (2008).

redshifts. Clearly, deep and wide observations with WFC3 will be able to disentangle the various scenarios for the evolution of the UV LF.

\section{Summary and conclusions}

We present in this work the results of a $Y$-band survey of the GOODS-South field, aimed at detecting galaxies at $\gtrsim 6.5$ and measuring their number density. With this purpose we made use of the deep $Y$-band observations of the GOODS-South field obtained with Hawk-I, the new near-IR camera installed at the VLT. We matched and combined these data with the publicly available images in the BVIZ (ACS), $U$ and $R$ (VIMOS), and $J H K$ (ISAAC) bands. The final area covered by these observations is of about $90 \operatorname{arcmin}^{2}$ at a magnitude limit $Y \simeq 26.7$.

We analysed this sample to select high-redshift $(z \gtrsim 6)$ candidate galaxies following a Lyman-break colour criterion adapted to our filter set. Galaxies are selected in the $Y$ band, and identified by their large colour break $Z-Y>1$. Particular care was taken in removing interlopers of various origins, including lower redshift galaxies with large Balmer breaks, variable sources, and Galactic brown dwarfs, although some residual contamination from the latter cannot be excluded. An additional class of interlopers, exhibiting large $Z-Y$ colours, as well as significant emission in the blue bands was removed by requiring a stringent non-detection in the UBVRI bands. We argue that some of these contaminants might be very faint emission-line galaxies or AGNs at intermediate redshift. The accuracy and statistical impact of these criteria were evaluated with extensive Monte Carlo imaging simulations.

From the same simulations we estimate that our redshift selection function is mostly efficient in the interval $6.4 \lessgtr z \lessgtr 7.2$. We eventually isolated 7 highly reliable $z$-drop candidates after removing from the colour selected sample one known galactic cool dwarf star and one source undetected in deep available NICMOS F160W images.

To estimate the constraints that our observations set on the evolution of the UV LF at $z>6.5$, we ran detailed and realistic imaging simulations of galaxy populations following different UV Schechter functions with linearly evolving parameters $\log (\phi)$ and $M_{*}$ and a fixed value $\alpha=-1.71$. Our simulations account for all the uncertainties involved in the observations: detection completeness, photometric scatter, and random fluctuations in the $S / N$ measure due to overlapping unresolved sources, 
or other effects. We compare the resulting distributions of simulated magnitudes and colours with the observed ones following a maximum likelihood approach to constrain the parameters of the evolving UV LF.

We find strong evidence of evolution of the LF above $z=6$ : our analysis rules out at a $\sim 99 \%$ confidence level that the LF remains constant in both $\phi$ and $M_{*}$ above $z=6$, even considering the effect of cosmic variance.

From our maximum likelihood analysis, we estimate $\Phi^{*}=$ $0.35_{-0.11}^{+0.16} \times 10^{-3} \mathrm{Mpc}^{-3}$ and $M^{*}(1500 \AA)=-20.24 \pm 0.45$ at the median redshift of our sample $(z=6.8)$. With respect to the values found at $z=6$, our best fit model indicates an evolution both in the normalisation factor $\phi$ and in the characteristic magnitude $M_{*}$. Our results are consistent with the recent analysis by Ouchi et al. (2009), considering the large statistical errors and the high degeneracy between the two parameters.

Fortunately, the uncertainty and the degeneracy in the $M_{*}$ and $\phi$ best-fit values are not reflected in a comparable uncertainty in the number density of bright galaxies, i.e. in the integral of the LF up to $M_{*}$. We derive a UV luminosity density $\rho_{\mathrm{UV}}=1.5_{-0.9}^{+2.0} \times$ $10^{25} \mathrm{erg} \mathrm{s}^{-1} \mathrm{~Hz}^{-1} \mathrm{Mpc}^{-3}(M<-19)$ and a star formation rate density $S F R D=2.8_{-1.6}^{+2.7} \times 10^{-3} M_{\text {sun }} \mathrm{yr}^{-1} \mathrm{Mpc}^{-3}$. These values are definitely lower than the corresponding ones at $z \sim 6$ by a factor $\sim 3.5$.

Although we did our best to carefully evaluate the systematic effects, we caution that our results depend on an extensive set of simulations to address the competing systematic effects that may increase or decrease the observed number of candidates. We note that the two most obvious - the possible residual contamination due to brown dwarfs and the possibility of a spurious detection for two of our candidates - will increase the amount of evolution from $z=6$ to $z=6.8$. Clearly, only spectroscopic surveys and/or deeper imaging in the IR will definitely settle the issue. Such strong evolution in the UV LF has strong consequences for reionisation scenarios, as well as for planning future surveys aimed at detecting very high-redshift galaxies and reionisation sources.

Determining whether the UV emission of normal galaxies is capable of reionising the Universe at $z>6$ requires knowledge of the value of many parameters that are still unconstrained at these redshifts: the escape fraction of ionising photons, the HII clumping factor, the exact spectrum of star forming galaxies, their dust content, as well as the shape of the stellar initial mass function and the metallicity of stellar populations (e.g. Madau et al. 1999; Barkana \& Loeb 2001; Stiavelli et al. 2004). In agreement with Bolton \& Haehnelt (2007) and their conclusions on the early estimates from Bouwens et al. (2005), the strong decrease we observe in the UV emission coming from relatively bright sources implies that this population by itself is not capable of reionising the Universe beyond redshift 6 . These observations can be reconciled with a completion of reionisation before $z=6$ only under the hypothesis of an evolution of the physical parameters quoted above, like an increase in the escape fraction, harder UV spectrum, a lower clumpiness factor, or lower metallicities (e.g. Henry et al. 2009; Oesch et al. 2009). Another possibility is that a relevant contribution to the UV emission comes from galaxies at the faint end of the LF (Bouwens et al. 2007) or from more exotic sources (see e.g. Venkatesan et al. 2003; Madau et al. 2004).

Thus it will be possible to fully assess the role of LBGs in the reionisation of the neutral IGM only putting tighter constraints both on the bright and on the faint end of the LF, at $z \sim 7$ and beyond.
Our results also have implications for the efficiency of these future surveys. We show that, to collect sizeable samples of $z>7$ bright galaxies, it is necessary to reach very faint limits $\left(m_{\text {F160W }} \gtrsim 28\right)$ over relatively large areas. On the other hand, the extrapolation of our results to higher redshifts indicates that dedicated surveys with HST-WFC3 will be able to disentangle the various scenarios for the evolution of the UV LF parameters above $z \sim 6$.

Acknowledgements. Observations were carried out using the Very Large Telescope at the ESO Paranal Observatory under Programme IDs LP181.A0717, LP168.A-0485, ID 170.A-0788, and the ESO Science Archive under Programme IDs 64.O-0643, 66.A-0572, 68.A-0544, 164.O-0561, 163.N-0210, and 60.A-9120.

\section{References}

Barkana, R., \& Loeb, A. 2001, Phys. Rep., 349, 125

Beckwith, S. V. W., Stiavelli, M., Koekemoer, A. M., et al. 2006, AJ, 132, 1729 Bertin, E., \& Arnouts, S. 1996, A\&AS, 117, 393

Bolton, J. S., \& Haehnelt, M. G. 2007, MNRAS, 382, 325

Bouwens, R. J., \& Illingworth, G. D. 2006, Nature, 443, 189

Bouwens, R. J., Illingworth, G. D., Rosati, P., et al. 2003, ApJ, 595, 589

Bouwens, R. J., Thompson, R. I., Illingworth, G. D., et al. 2004, ApJ, 616, L79

Bouwens, R. J., Illingworth, G. D., Thompson, R. I., \& Franx, M. 2005, ApJ, 624, L5

Bouwens, R. J., Illingworth, G. D., Blakeslee, J. P., \& Franx, M. 2006, ApJ, 653, 53

Bouwens, R. J., Illingworth, G. D., Franx, M., \& Ford, H. 2007, ApJ, 670, 928 Bouwens, R. J., Illingworth, G. D., Franx, M., \& Ford, H. 2008, ApJ, 686, 230 Bouwens, R. J., Illingworth, G. D., Bradley, L. D., et al. 2009, ApJ, 690, 1764 Bouwens, R. J., Illingworth, G. D., Oesch, P. A., et al. 2010, ApJ, 709, L69 Bradley, L. D., Bouwens, R. J., Ford, H. C., et al. 2008, ApJ, 678, 647 Bruzual, A. G. 2007a, in IAU Symp. 241, ed. A. Vazdekis, \& R. F. Peletier, 125

Bruzual, G. 2007b, in From Stars to Galaxies: Building the Pieces to Build Up the Universe, ed. A. Vallenari, R. Tantalo, L. Portinari, \& A. Moretti, ASP Conf. Ser., 374, 303

Bunker, A. J., Stanway, E. R., Ellis, R. S., \& McMahon, R. G. 2004, MNRAS, 355,374

Bunker, A., Wilkins, S., Ellis, R., et al. 2009, MNRAS, in press [arXiv: 0909.2255]

Burgasser, A. J. 2004, ApJS, 155, 191

Burgasser, A. J., Kirkpatrick, J. D., Brown, M. E., et al. 1999, ApJ, 522, L65

Burgasser, A. J., Geballe, T. R., Leggett, S. K., Kirkpatrick, J. D., \& Golimowski, D. A. 2006, ApJ, 637, 1067

Calzetti, D., Armus, L., Bohlin, R. C., et al. 2000, ApJ, 533, 682

Casali, M., Pirard, J.-F., Kissler-Patig, M., et al. 2006, in SPIE Conf. Ser., 6269

Chabrier, G., Baraffe, I., Allard, F., \& Hauschildt, P. H. 2005

[arXiv: astro-ph/0509798]

D’Antona, F., Oliva, E., \& Zeppieri, A. 1999, A\&A, 352, 567

Delorme, P., Willott, C. J., Forveille, T., et al. 2008, A\&A, 484, 469

Dickinson, M., Stern, D., Giavalisco, M., et al. 2004, ApJ, 600, L99

Dow-Hygelund, C. C., Holden, B. P., Bouwens, R. J., et al. 2007, ApJ, 660, 47

Giavalisco, M., Dickinson, M., Ferguson, H. C., et al. 2004, ApJ, 600, L103

Grazian, A., Fontana, A., de Santis, C., et al. 2006, A\&A, 449, 951

Helling, C., Ackerman, A., Allard, F., et al. 2008, MNRAS, 391, 1854

Henry, A. L., Siana, B., Malkan, M. A., et al. 2009, ApJ, 697, 1128

Hickey, S., Bunker, A., Jarvis, M. J., Chiu, K., \& Bonfield, D. 2009, MNRAS, in press [arXiv:0909.4205]

Hu, E. M., Cowie, L. L., Kakazu, Y., \& Barger, A. J. 2009, ApJ, 698, 2014

Iwata, I., Ohta, K., Tamura, N., et al. 2007, MNRAS, 376, 1557

Iye, M., Ota, K., Kashikawa, N., et al. 2006, Nature, 443, 186

Izotov, Y. I., Papaderos, P., Guseva, N. G., Fricke, K. J., \& Thuan, T. X. 2004,

A\&A, 421, 539

Izotov, Y. I., Thuan, T. X., \& Guseva, N. G. 2007, ApJ, 671, 1297

Kissler-Patig, M., Pirard, J.-F., Casali, M., et al. 2008, A\&A, 491, 941

Kitzbichler, M. G., \& White, S. D. M. 2007, MNRAS, 376, 2

Leggett, S. K., Geballe, T. R., Fan, X., et al. 2000, ApJ, 536, L35

Luo, B., Bauer, F. E., Brandt, W. N., et al. 2008, ApJS, 179, 19

Madau, P. 1995, ApJ, 441, 18 
A\&A 511, A20 (2010)

Madau, P., Pozzetti, L., \& Dickinson, M. 1998, ApJ, 498, 106 Madau, P., Haardt, F., \& Rees, M. J. 1999, ApJ, 514, 648

Madau, P., Rees, M. J., Volonteri, M., Haardt, F., \& Oh, S. P. 2004, ApJ, 604, 484

Mannucci, F., Buttery, H., Maiolino, R., Marconi, A., \& Pozzetti, L. 2007, A\&A, 461,423

McLure, R. J., Cirasuolo, M., Dunlop, J. S., Foucaud, S., \& Almaini, O. 2009, MNRAS, 395, 2196

McLure, R. J., Dunlop, J. S., Cirasuolo, M., et al. 2010, MNRAS, in press [arXiv:0909.2437]

Meurer, G. R., Heckman, T. M., \& Calzetti, D. 1999, ApJ, 521, 64

Nonino, M., Dickinson, M., Rosati, P., et al. 2009, ApJS, 183, 244

Oesch, P. A., Carollo, C. M., Stiavelli, M., et al. 2009, ApJ, 690, 1350

Oesch, P. A., Bouwens, R. J., Illingworth, G. D., et al. 2010, ApJ, 709, L16

Ouchi, M., Shimasaku, K., Okamura, S., et al. 2004, ApJ, 611, 660

Ouchi, M., Mobasher, B., Shimasaku, K., et al. 2009, ApJ, 706, 1136

Overzier, R. A., Guo, Q., Kauffmann, G., et al. 2009, MNRAS, 394, 577

Patten, B. M., Stauffer, J. R., Burrows, A., et al. 2006, ApJ, 651, 502

Pinfield, D. J., Burningham, B., Tamura, M., et al. 2008, MNRAS, 390, 304

Pirard, J.-F., Kissler-Patig, M., Moorwood, A., et al. 2004, in SPIE Conf. Ser. 5492, ed. A. F. M. Moorwood, \& M. Iye, 1763

Reddy, N. A., \& Steidel, C. C. 2009, ApJ, 692, 778

Richard, J., Pelló, R., Schaerer, D., Le Borgne, J.-F., \& Kneib, J.-P. 2006, A\&A, 456,861
Richard, J., Stark, D. P., Ellis, R. S., et al. 2008, ApJ, 685, 705

Ryan, Jr., R. E., Hathi, N. P., Cohen, S. H., \& Windhorst, R. A. 2005, ApJ, 631, L159

Santini, P., Fontana, A., Grazian, A., et al. 2009, A\&A, 504, 751

Sawicki, M., \& Thompson, D. 2006, ApJ, 648, 299

Schechter, P. 1976, ApJ, 203, 297

Shimasaku, K., Ouchi, M., Furusawa, H., et al. 2005, PASJ, 57, 447

Stanway, E. R., Bunker, A. J., \& McMahon, R. G. 2003, MNRAS, 342, 439

Stanway, E. R., Bremer, M. N., \& Lehnert, M. D. 2008a, MNRAS, 385, 493

Stanway, E. R., Bremer, M. N., Squitieri, V., Douglas, L. S., \& Lehnert, M. D. 2008b, MNRAS, 386, 370

Steidel, C. C., Pettini, M., \& Hamilton, D. 1995, AJ, 110, 2519

Steidel, C. C., Adelberger, K. L., Giavalisco, M., Dickinson, M., \& Pettini, M. 1999, ApJ, 519, 1

Stiavelli, M., Fall, S. M., \& Panagia, N. 2004, ApJ, 610, L1

Trenti, M., \& Stiavelli, M. 2008, ApJ, 676, 767

Venkatesan, A., Tumlinson, J., \& Shull, J. M. 2003, ApJ, 584, 621

Vanzella, E., Giavalisco, M., Dickinson, M., et al. 2009, ApJ, 695, 1163

Wilkins, S. M., Bunker, A. J., Ellis, R. S., et al. 2009, MNRAS, in press [arXiv: 0910.1098]

Yan, H., Windhorst, R., Hathi, N., et al. 2009, ApJ, submitted [arXiv:0910.0077]

Yoshida, M., Shimasaku, K., Kashikawa, N., et al. 2006, ApJ, 653, 988 Zheng, W., Bradley, L. D., Bouwens, R. J., et al. 2009, ApJ, 697, 1907 